\title{
Spectral properties of asteroids in cometary orbits
}

\author{
J. Licandro ${ }^{1}$, A. Alvarez-Candal ${ }^{2}, \star$, J. de León ${ }^{1}$, N. Pinilla-Alonso ${ }^{3}$, D. Lazzaro ${ }^{2}$, and H. Campins ${ }^{4,5}$ \\ 1 Instituto de Astrofísica de Canarias, c/Vía Láctea s/n, 38205, La Laguna, Tenerife, Spain \\ e-mail: jlicandr@iac.es \\ 2 Observatório Nacional - MCT. Rua Gal. José Cristino 77, Rio de Janeiro, 20921-400 RJ, Brazil \\ 3 Fundación Galileo Galilei \& Telescopio Nazionale Galileo, PO Box 565, 38700, S/C de La Palma, Tenerife, Spain \\ ${ }^{4}$ Physics Department, University of Central Florida, Orlando, FL, 32816, USA \\ 5 LPL, University of Arizona, Tucson Az, 85721, USA
}

Received 23 July 2007 / Accepted 18 December 2007

\section{ABSTRACT}

\begin{abstract}
Aims. In this paper we analyze the spectra of a sample of asteroids in cometary orbits (ACOs) in order to understand the relationship between them, the Jupiter family comets (JFCs), and the outer main belt populations, such as Hilda, Trojan and Cybele asteroids. Methods. We obtained visible $(0.55-0.90 \mu \mathrm{m})$ and/or near-infrared $(0.8-2.3 \mu \mathrm{m})$ spectra of 24 ACOs using 3 telescopes at the "Roque de los Muchachos" Observatory (La Palma, Spain). Using this data, we derived the taxonomic classification of the asteroids. As most ACOs present featureless spectra (B-, C-, P-, D-type) we also derived their spectral gradient $\left(S^{\prime}\right)$. Considering also published spectra of ACOs we correlated $S^{\prime}$ with orbital and dynamical parameters and obtained the cumulative distribution of $S^{\prime}$, and compare it with that of other related populations.

Results. We present visible and/or near infrared spectra of 24 ACOs, most of them (21) presenting featureless spectra. After including the spectra of other ACOs already published, we analyzed a total of 41 objects, 34 of them having featureless spectra like the spectra of comet nuclei and outer main-belt asteroids. We also noticed a significant difference in the taxonomic distribution of the ACOs in Near-Earth orbits $(q<1.3 \mathrm{AU})$ and those that are not $(q>1.3 \mathrm{AU})$, indicative of different source/transport mechanisms. About $35 \%$ of the ACOs in the NEO population analyzed have spectra that present the typical silicate absorption bands at 1 and $2 \mu \mathrm{m}$ (S- and V-type), while only 1 of the 24 ACOs in the non-NEO population (about 4\%) is S-type and the other 23 have a featureless spectrum. Thus the NEO sub-population of ACOs is composed of a significant fraction of asteroids scattered from the inner main-belt. We didn't find any subtle features in the $0.5-2.0 \mu \mathrm{m}$ spectral region of featureless ACOs that can be used to discriminate wheather an ACO comes from a cometary or an asteroidal origin. The analysis of the spectral gradient shows that ACOs present an interesting, and significant, anti-correlation between the Tisserand parameter and the "spectral gradient", meaning that the reddest objects have the lower Tisserand parameter (i.e., higher chance of a cometary origin). Finally, we obtained the $S^{\prime}$ cumulative distribution for ACOs. This distribution is "bluer" than that of comet nuclei, Damocloids and outer main-belt population of asteroids, indicative of a significant "contamination" of asteroids scattered from the inner main-belt.
\end{abstract}

Key words. minor planets, asteroids - comets: general - methods: observational - techniques: spectroscopic

\section{Introduction}

Comets and asteroids used to be considered as two distinct classes of small bodies of the Solar System, both being remnants from the early stages of its formation. Comets are residual planetesimals formed in a region that extends from the giant planets to the limits of the solar nebula. Even if they are partially processed, comets (and other related icy minor planets, like transneptunian objects and Centaurs), are the most pristine observable Solar System objects. The present population of asteroids is the product of the collisional and dynamical evolution of remnant planetesimals formed in the region between Mars and Jupiter. As a consequence of the different formation regions, the volatile content of the two populations is different. The most apparent distinction between comets and asteroids is that comet nuclei, when close to the Sun, are surrounded by a coma produced by the outgassing of volatiles, while asteroids do not. So, up to now, a minor body has been considered a comet or an asteroid depending on the detection of a coma.

\footnotetext{
* Present address: LESIA, Observatoire de Paris, 92195 Meudon Principal Cedex, France.
}

From a dynamical point of view, the orbits of most comets and most asteroids are remarkably different. The criterion commonly used to differentiate between a cometary or an asteroidal orbit is related to the Tisserand parameter (Kresák 1979), defined as, $T=a_{J} / a+2 \cos I \sqrt{\left(a / a_{J}\right)\left(1-e^{2}\right)}$, where $a$ and $a_{J}$ are the semi-major axes of the orbits of the asteroid and Jupiter, respectively, while $e$ and $I$ are the eccentricity and the inclination of the asteroid's orbit. $I$ is measured relative to the orbital plane of Jupiter. Most comets have unstable orbits with $T<3$, while the great majority of asteroids have orbits with $T>3$. Only a few known comets have $T$ slightly larger than 3, being the case of $2 \mathrm{P} /$ Encke the most remarkable, but this can be due to the long term effects of non-gravitational forces (Fernández et al. 2002).

The large number of asteroids discovered in the last two decades by different surveys, has demonstrated that there is a significant number of asteroids in comet-like orbits. At this point, the simple distinction between comets and asteroids starts to become insufficient. Other important discoveries show that the simple definition needs a revision: (1) the discovery of objects in asteroidal orbits that have temporal bursts of activity e.g. (4015) Wilson-Harrington and (7968) Elst-Pizarro; (2) the discovery of asteroidal objects that have associated meteor showers that 
suggest some past cometary activity, e.g., (3200) Phaethon; (3) the discovery of icy objects (TNOs and Centaurs) that due to their distance from the Sun rarely develop a coma; (4) the realization that comets might develop an asteroidal appearance when sublimation stops, either due to the depletion of volatile materials (dead or extinct comets) or by the growth of a surface crust of refractory material up to a thickness that prevents subsurface volatiles from warming up to sublimation temperature (dormant comets, Rickman et al. 1990).

Objects in cometary orbits are dynamically unstable, implying that the population needs to be continually replenished. Therefore, there should exist some dynamical mechanism that perturbs the orbit of typical asteroids changing their Tisserand invariant from $T>3$ to $T<3$, and/or physical mechanism that deactivates comet nuclei, transforming a typical active comet into an asteroidal object. Therefore, all objects with $T<3$ and asteroidal aspect (without an observable coma) are called asteroids in cometary orbits (ACOs) and are candidates to be dead or dormant comets.

On the other hand, we consider objects in asteroidal orbits with intermittent or past cometary-like activity as "activated asteroids" (AAs). The recent discovery of a population of AAs in the main belt (Hsieh \& Jewitt 2006) and their similarities with Phaethon-like objects (Licandro et al. 2007), suggest that these two populations are the same kind of objects, probably asteroids with some ice that remains in their interior. The activity in AAs may be triggered by impacts with small bodies that expose this ice to the Sun.

ACOs and AAs are objects in the transition zone between asteroids and comets, and their study is very important from a cosmogonical point of view. In particular, the study of ACOs is very important to understand the formation of cometary dust mantles and the end states of comets, in order to determine the population of Jupiter family comets (JFCs), and to understand the dynamical processes that move asteroids from typical asteroidal orbits to cometary-like ones.

We will restrict the following analysis to objects with $2 \leq$ $T \leq 3.02$, and $q<5 \mathrm{AU}$, which is slightly more restrictive than the common range of Tisserand parameter of JFCs (Tancredi et al. 2006). Dead or dormant comets in this restricted population should come from the JFC, and the physical properties of this population are better known than that of other comets. Asteroidal objects with $T<2$ are usually called Damocloids (Jewitt 2005) and are asteroids in Halley-type cometary orbits. The properties of Damocloids will not be discussed here due to the small sample of known objects. It is also worth to note that objects such as Trojan, Hilda or Cybele asteroids are excluded from the ACO population, even though some of them have $T<3.02$.

Hartmann et al. (1987) suggested that active, dormant and dead comets are very dark often reddish, objects, with spectra similar to D-, P- and C-type asteroids of the outer Solar System with albedos and color probably controlled by carbonaceous dust containing reddish organic compounds.

In recent years some studies have searched for evidence that could help identifying whether an ACO comes from an asteroidal or a cometary source. In Licandro et al. (2006) we presented a preliminary analysis of a sub-sample of the visible and near-infrared spectra presented in this paper. We found $15 \mathrm{ob}-$ jects with featureless spectra and $2 \mathrm{~S}$-type asteroids, we did not find any subtle feature that can be used to discriminate between D-type main belt asteroids and ACOs with featureless spectra, we noted that the reflectivity gradient distribution of ACOs and comet nuclei seems different, although much more spectroscopic work is needed to improve these distributions. Also in
Licandro et al. (2006), by dividing ACOs in two populations, NEO (objects with $q \leq 1.3 \mathrm{AU}$ ) and non-NEO (objects with $q>1.3 \mathrm{AU}$ ), we noticed that the first population is composed of a significant number of S or Q-type asteroids ( 2 of the $3 \mathrm{ob}-$ jects presented in that paper and 5 of the 16 objects observed by Binzel et al. (2004), while the non-NEO ACOs population is composed mainly of objects with featureless spectra, and concluded that this spectral difference suggests a different origin for these two populations.

In Alvarez-Candal \& Licandro (2006) we studied the cumulative size distribution (CSD) of the ACOs and found a suggestive difference in the indeces of the cumulative size distribution between the ACOs in near Earth orbits and those that are not. The latter have an index similar to that reported for the Jupiter family comets (Tancredi et al. 2006), while the former have index similar to those of the outer belt populations.

Fernandez et al. (2005) analyzed the albedo distribution of a sample of asteroids in cometary orbits, finding that, in general they have lower albedos than objects with $T>3$. Moreover, they found that, for ACOs with $T<2.6$, all of them have $p_{V}<$ 0.075 , i.e., similar to those measured for cometary nuclei, and suggested a cometary origin.

Nevertheless, no definitive discriminators have resulted from those works. The only secure information is that comets present neutral or red featureless spectra. Most of them have spectra similar to that of P- or D-type asteroids (Jewitt 2002; Licandro et al. 2002b; Campins et al. 2006) and have very low albedos (Fernandez et al. 2005). Therefore, the aim of this work is to compare the spectral properties of ACOs with those of asteroids from populations possibly related to cometary sources, such as Trojan and Hilda asteroids, and with other main belt D-type asteroids (Cybeles and D-types with semi-major axis $a<3.2 \mathrm{AU}$ ). Our aim is to sear for spectral features (e.g. an absorption band) that could be used to discriminate between a cometary and an asteroidal nature, and to obtain statistical properties of the different populations that could help to determine the sources of ACOs.

In the following section we present the new observations and in Sect. 3 the taxonomic analysis. In Sects. 4 and 5 we describe a quantitative analysis of the spectral slopes, discuss any correlation with dynamical parameters, and we compare the ACOs with related populations of asteroids and JFCs. The main results are summarized in Sect. 6.

\section{Observations}

Visible and/or near-infrared spectra of 24 ACOs covering the $0.5-2.4 \mu \mathrm{m}$ spectral region were obtained during different nights as part of an observational campaign that started in 2002 using three different telescopes of the "Roque de los Muchachos Observatory" (Canary Islands, Spain). The list of observed objects and observational circumstances are shown in Table 1. Also, 1 Trojan, 5 Hilda and 4 D-type inner main belt asteroids (with semi-major axes $a<3.2 \mathrm{AU}$ ) were observed in order to have a comparison sample. Trojan and Hilda asteroids are representative of the outer main belt population and could be sources of JFCs (Levison \& Duncan 1997; Kresák 1979; di Sisto et al. 2005). According to Fernández et al. (2002), the outer main belt could be the only source of ACOs in the NEA population, thus a comparison with the spectral properties of outer main belt asteroids is desirable. The outer main belt is dominated by Dtype asteroids, so comparison with D-Type objects of the inner main belt is also important to search for spectral differences that could help to identify the sources of ACOs. Notice also that most 


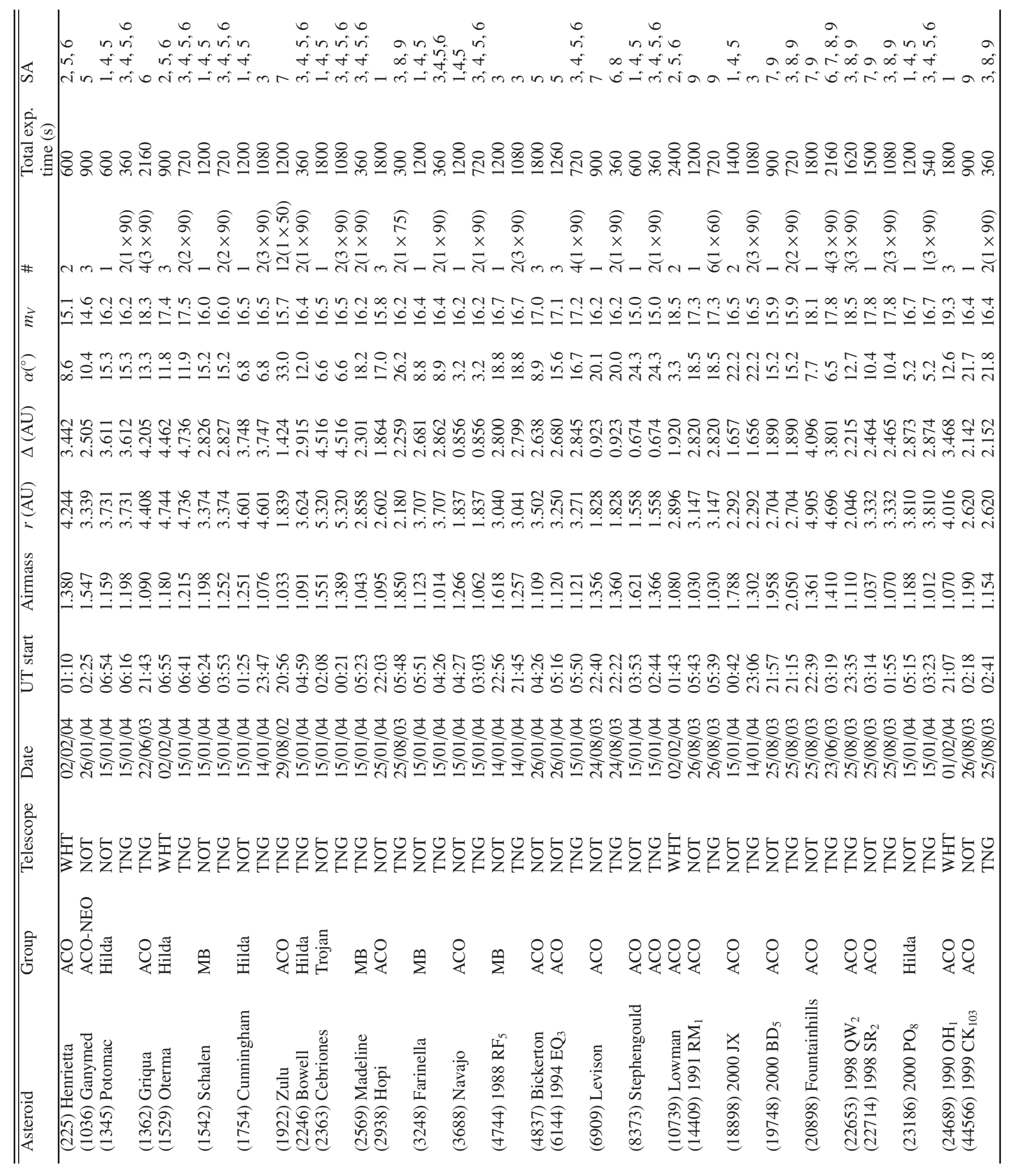






observed comet nuclei present a D-type like spectrum in the visible and near-infrared (e.g. Campins et al. 2006).

Low resolution near-infrared spectra were taken with the $3.56 \mathrm{~m}$ Telescopio Nazionale Galileo (TNG) using the low resolution mode of NICS (Near Infrared Camera Spectrograph), based on an Amici prism disperser that covers the $0.8-2.4 \mu \mathrm{m}$ region (Oliva 2000). The slit was oriented in the parallactic angle, and the tracking was at the asteroid proper motion. The width of the slit used was $1.5^{\prime \prime}$ and corresponds to a spectral resolving power $R \approx 34$ quasi-constant along the spectra. The observational method and reduction procedure followed Licandro et al. (2002a). The acquisition consisted of a series of short exposure images in one position of the slit (position $A$ ) and then offsetting the telescope by $10^{\prime \prime}$ in the direction of the slit (position $B$ ), and obtaining another series of images. This process was repeated and a number of $A B B A$ cycles were acquired. The total on-object exposure time is listed in Table 1. The two-dimensional spectra were extracted, and collapsed to one dimension. The wavelength calibration was performed using a look-up table which is based on the theoretical dispersion predicted by ray-tracing and adjusted to best fit the observed spectra of calibration sources and telluric absorptions. To correct for telluric absorption and to obtain the relative reflectance, several G2 stars from the list of Landolt (1992) were observed during the same night at airmasses similar to that of the asteroids (see Table 1). These Landolt stars were also observed on previous nights together with the solar analogue star P330E (Colina \& Bohlin 1997) and they show similar spectra in the infrared region, so we used them as solar analogues.

Finally, the spectra of the asteroids were divided by the spectra of the solar analogue stars observed the same night, and the so obtained reflectance spectra averaged, obtaining the final relative reflectance spectrum of each object. Sub-pixel offsetting was applied when dividing the two spectra to correct for errors in the wavelength calibrations due to instrumental flexure. By comparing the reflectance spectra of the same asteroid obtained with different solar analogues we determined that the uncertainty in the slope is smaller than $1 \% / 0.1 \mu \mathrm{m}$.

Visible spectra were obtained with the $2.5 \mathrm{~m}$ Nordic Optical Telescope (NOT) using ALFOSC and with the $4.2 \mathrm{~m}$ William Herschel telescope (WHT) using the red arm of ISIS spectrograph. A grism disperser that covers the $0.32-0.91 \mu \mathrm{m}$ with a dispersion of $3 \AA /$ pixel, and a second order blocking filter that cut at $0.495 \mu \mathrm{m}$ were used with ALFOSC. The R158R grating centered at 7.500 Åwas used in ISIS covering the $0.50-0.92 \mu \mathrm{m}$ region with a dispersion of $1.63 \AA$ /pixel. A $1.3^{\prime \prime}$ and a $2^{\prime \prime}$ slit width were used with ALFOSC and ISIS respectively. The slit was oriented in the parallactic angle, and the tracking was at the asteroid proper motion.

Three spectra of each object were obtained by shifting the object $5^{\prime \prime}$ in the slit direction to better correct the fringing. Visible data reduction was done using standard IRAF procedures. Images were over-scan and bias corrected, and flat-field corrected using lamp flats. The two-dimensional spectra were extracted, sky background subtracted, and collapsed to one dimension. The wavelength calibration was done using the Neon and Argon lamps. The three spectra of each asteroid were averaged. The reflectance spectra were obtained by dividing the spectra of the asteroids by the spectra of different solar analogue stars and/or G2 stars from Landolt (1992), observed during the same night at airmasses similar to those of the asteroids (see Table 1). 


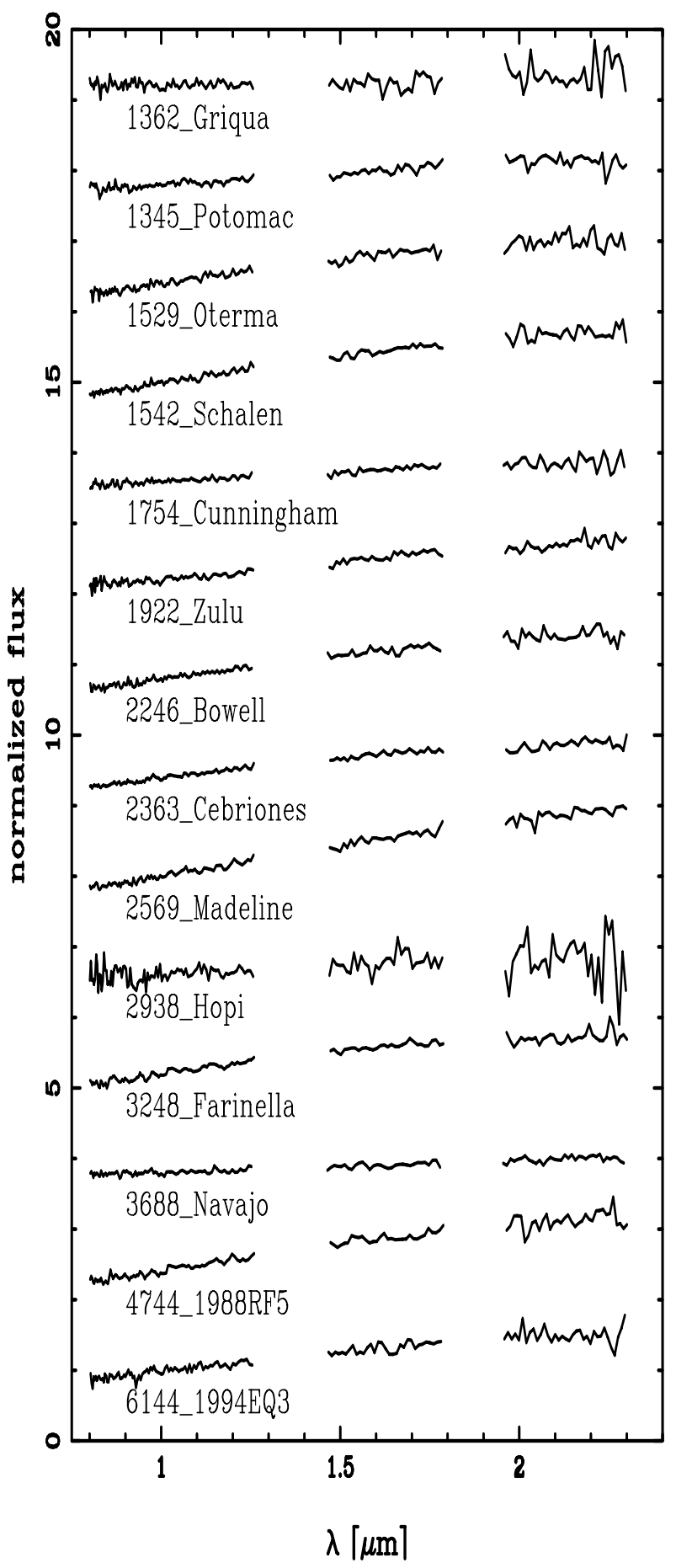

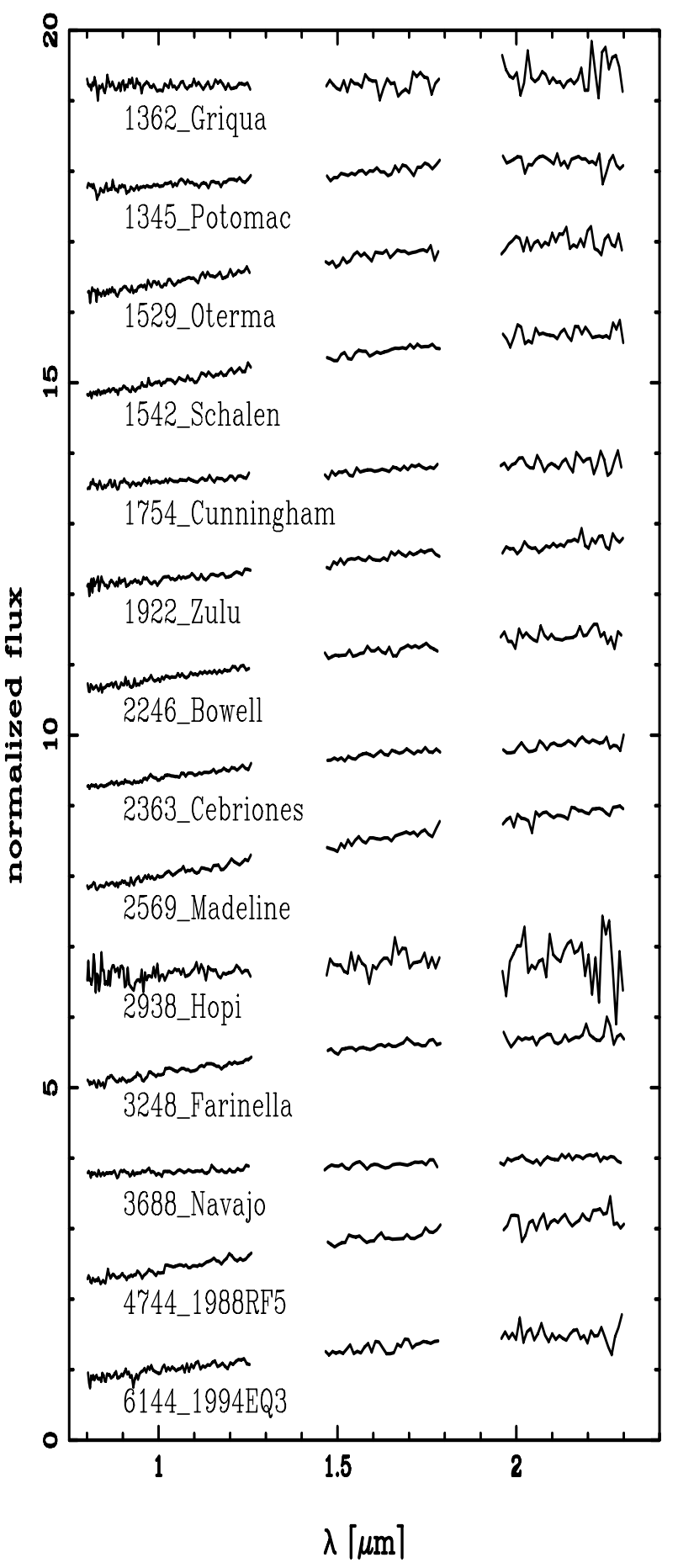

Fig. 1. Near-infrared reflectance spectra of the asteroids, normalized at $1.0 \mu \mathrm{m}$. Spectra are shifted in reflectance for clarity.

The final reflectance spectra in the visible region are shown in Fig. 2 while the NIR spectra are shown in Fig. 1. In these figures visible spectra are normalized at $0.6 \mu \mathrm{m}$, and NIR spectra are normalized at $1.0 \mu \mathrm{m}$.

\section{Taxonomic analysis}

Taxonomic classification can help to discriminate the nature of an asteroid providing information on the surface composition. We use the taxonomy based on the visible $(0.3-1.1 \mu \mathrm{m})$ spectrum defined by Tholen \& Barucci (1989).
We divide the taxonomic classes in two groups, those that present typical silicate absorption bands around 1 and $2 \mu \mathrm{m}$ in the spectrum like S- Q- A- and V-type asteroids, and those without features in the $0.5-2.5 \mu \mathrm{m}$ spectral region, the B-, C-, X (P)-, $\mathrm{D}$-types asteroids with a spectrum characterized by their linear trend and lack of strong absorption features. Henceforth we will call the first group of objects "with bands" and the second group "featureless".

From previous works (Gradie et al.1989; Mothé-Diniz et al. 2003), we know that asteroids with bands usually predominate in the inner main belt region and are less abundant in the outer main belt. On the other hand, featureless asteroids are mostly 

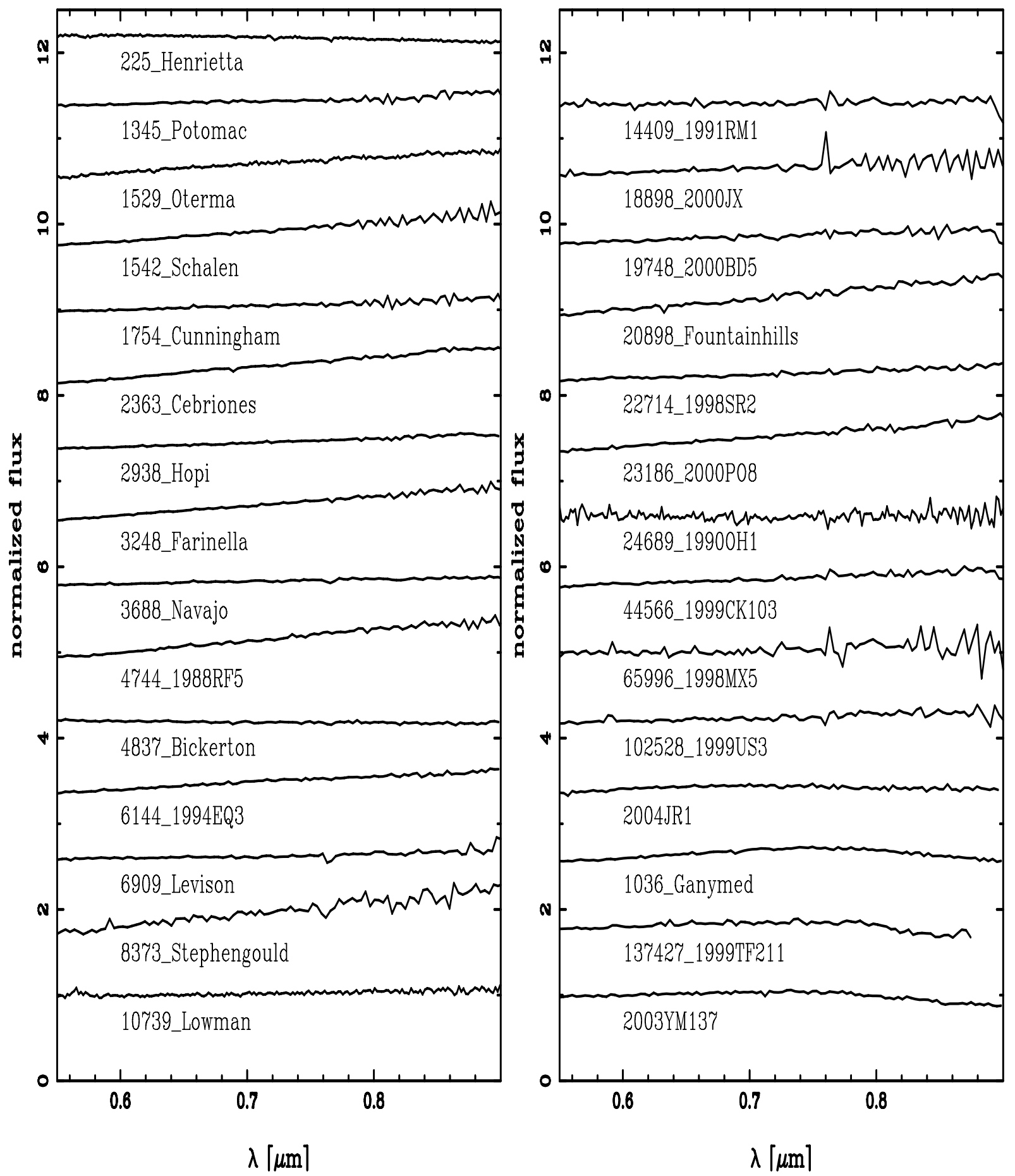

Fig. 2. Visible reflectance spectra of the asteroids, normalized at $0.6 \mu \mathrm{m}$. Spectra are shifted in reflectance for clarity.

associated with outer main belt populations like Hilda, Cybele, and Trojan asteroids, and icy bodies such as comet nuclei. In particular, all the published spectra of comet nuclei are similar to the X (P)- and D-type asteroids (e.g. Jewitt 2002; Licandro et al. 2002b; Campins et al. 2006). Any asteroidal object with SQ- and V-type spectra unlikely have a cometary origin since the mineralogy associated with these taxonomic types is igneous or that of the ordinary chondrites.

We started the analysis of the spectra by determining their taxonomic classification. The visible spectra are used to classify the objects by comparison with templates from the Tholen and Barucci classification scheme (Tholen 1984;
Tholen \& Barucci 1989). The taxonomic classification of the ACOs observed as well as that taken from the literature, is given in Table 2.

Most of the observed ACOs have featureless spectra. Spectra range from slightly bluish, B-type, to red, D-type, objects. Few exceptions are observed: two S-type, (1036) Ganymed and 1999 $\mathrm{TF}_{211}$, and a V-type $2003 \mathrm{YM}_{137}$. The first of these was already reported as an S-type (Tholen \& Barucci 1989; Mothé-Diniz et al. 2000) and its near-infrared spectrum was also measured by Bell et al. (1988).

In order to increase our database we also searched for spectra of ACOs in the major visible spectroscopic surveys, the S3OS2 
Table 2. Taxonomic classification of the ACOs observed in this work and ACOs taken from the literature, as defined by Tholen \& Barucci (1989).

\begin{tabular}{|c|c|c|c|}
\hline Object & Tax. & Object & Tax. \\
\hline (225) Henrietta & $\mathrm{B}$ & $(22714) 1998 \mathrm{SR}_{2}$ & $\mathrm{D}$ \\
\hline (692) Hippodamia & $\mathrm{S}$ & (24689) $1990 \mathrm{OH}_{1}$ & $\mathrm{C}$ \\
\hline (1036) Ganymed & $S$ & (44566) $1999 \mathrm{CK}_{103}$ & D \\
\hline (1362) Griqua & B & (65996) $1998 \mathrm{MX}_{5}$ & $\mathrm{P}$ \\
\hline (1373) Cincinnati & $\mathrm{D}$ & (85490) $1997 \mathrm{SE}_{5}$ & D \\
\hline (2906) Caltech & $\mathrm{P}$ & (137427) $1999 \mathrm{TF}_{211}$ & $\mathrm{~S}$ \\
\hline (2938) Hopi & $\mathrm{D}$ & ${ }^{(102528)} 1999 \mathrm{US}_{3}$ & D \\
\hline (3552) Don Quixote & D & 1992 UB & D \\
\hline (3688) Navajo & $\mathrm{P}$ & $1999 \mathrm{SE}_{10}$ & D \\
\hline (4837) Bickerton & $\mathrm{C}$ & $1999 \mathrm{DB}_{2}$ & $\mathrm{~S}$ \\
\hline (4997) Ksana & B & $2000 \mathrm{NM}$ & V \\
\hline (6144) $1994 \mathrm{EQ}_{3}$ & $\mathrm{D}$ & $2000 \mathrm{PG}_{3}$ & $\mathrm{D}$ \\
\hline (6909) Levison & $\mathrm{P}$ & $2000 \mathrm{WL}_{10}$ & $\mathrm{C}$ \\
\hline (7604) Kridsadaporn & $\mathrm{C}$ & $2001 \mathrm{EC}$ & V \\
\hline (8373) Stephengould & D & $2001 \mathrm{TX}_{16}$ & $\mathrm{D}$ \\
\hline (10739) Lowman & $\mathrm{P}$ & $2001 \mathrm{UC}_{5}$ & $\mathrm{D}$ \\
\hline (14409) $1991 \mathrm{RM}_{1}$ & $\mathrm{P}$ & $2001 \mathrm{UU}_{92}$ & D \\
\hline (18898) 2000 JX & D & $2003 \mathrm{YM}_{137}$ & V \\
\hline (19748) $2000 \mathrm{BD}_{5}$ & D & $2004 \mathrm{JR}_{1}$ & $\mathrm{C}$ \\
\hline (20898) Fountainhills & D & & \\
\hline
\end{tabular}

(Lazzaro et al. 2004 ${ }^{1}$ ) and the SMASS (Xu et al. 1995; Bus \& Binzel 2002 ${ }^{2}$ ). Most of the SMASS spectra of ACOs, a total of sixteen, belong to the NEO population (Binzel et al. 2004). Most of the spectra of ACOs from S3OS2 and SMASS are also featureless; only two of them being S-type, (692) Hippodamia and $1999 \mathrm{DB}_{2}$, and another two being V-type, $2000 \mathrm{NM}$ and 2001 EC. The final sample has 41 ACOs with observed spectra, 34 of them are featureless while 7 are "with bands" and thus unlikely have a cometary origin.

\subsection{Two sub-populations}

The larger sample presented in this work confirms the initial conclusions presented in Licandro et al. (2006): most ACOs with spectral bands belong to the NEO population (objects with perihelion distance $q<1.3 \mathrm{AU}$ ), while the non-NEO population of ACOs (objects with $q>1.3 \mathrm{AU}$ ) is almost devoid of asteroids with bands. The enlarged sample presented in this paper confirms this result, which is clearly seen in Fig. 3. The sample of ACOs belonging to the NEO population consist of 17 objects, 11 of them have featureless spectra and 6 with bands. On the other hand only one of the total sample of 24 ACOs belonging to the non-NEO population presents a spectrum with bands, (692) Hippodamia. As in Licandro et al. (2006) we conclude that, while the NEO sub-population of ACOs is composed by a large fraction, about $35 \%$, of asteroids with bands, the non-NEO sub-population is mainly composed of featureless objects.

The difference in the taxonomies observed between the two sub-populations of ACOs might be indicative of different sources. We recall that the orbits of ACOs are dynamically unstable since they are prone to close encounters with Jupiter, hence, they need to be continuously replenished. Therefore, there should exist some dynamical mechanisms that perturb the orbit of typical asteroids changing their Tisserand invariant from $T>3$ to $T<3$, or some physical mechanisms that deactivate comet nuclei transforming an active comet into an object with

\footnotetext{
${ }^{1}$ http://www.psi.edu/pds/s3os2.html

${ }^{2}$ http://smass.mit.edu/
}

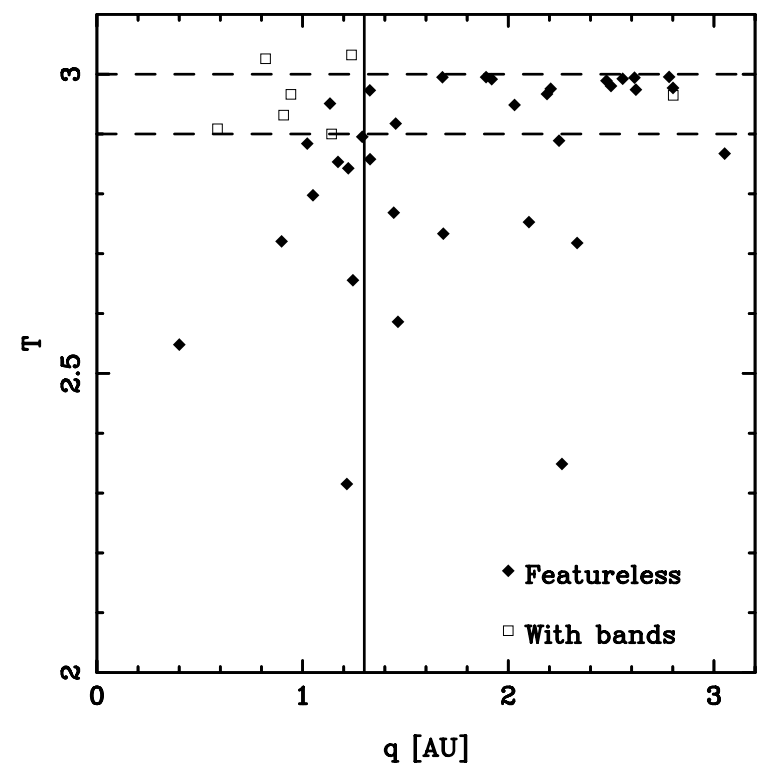

Fig. 3. Plot for ACOs in the perihelion distance $(q)$ - Tisserand parameter space. Open squares indicate spectra with bands and filled rhombi indicate featureless spectra. The vertical line separates NEO $(q \leq 1.3) \mathrm{AU}$ and the non-NEO groups. The dashed lines indicate $T=3$ and $T=2.9$. Notice that all observed ACOs with $T<2.9$ are featureless, and most of the asteroids with bands are NEOs.

asteroidal appearance. The abundance of objects with bands in the NEO sub-population of ACOs seem to indicate that it is composed of a significant fraction of asteroids dynamically scattered from the inner main belt. On the other hand, the non-NEO subpopulation seems to be mostly replenished by dynamically scattered asteroids from the outer main belt and/or deactivated comet nuclei.

Notice also in Fig. 3 that all observed ACOs with $T<2.9$ are featureless, so the mechanism that drives inner main-belt asteroids to cometary orbits is only efficient in producing orbits with Tisserand very close to 3 , and thus the $T>2.9$ is "contaminated" with these main-belt interlopers.

\section{Search for subtle spectral features}

One of the objectives of this work is to search for subtle features that could help to discriminate whether an ACO comes either from a cometary or an asteroidal source. In Licandro et al. (2006) we compared a "mean" ACO spectrum, computed as the mean of the 7 highest signal-to-noise spectra of the sample, with a "mean" D-type spectrum computed as the mean of 5 high signal-to noise spectra of D-type asteroids not belonging to the ACO population. Nevertheless, to use the signal-to-noise as the only criterion to compute these mean spectra does not take into account that the mechanism affecting the surfaces depends on the diameter of the objects (see Luu \& Jewitt 1996; Gil-Hutton 2002; Thélbault \& Doressoundiram 2003). Therefore, in this paper we use, as a second criterion, that the objects must have a comparable absolute magnitude, which assures that the diameters are roughly similar. The comparison is made between the ACO and Hilda, Trojan, D-type, Cybele asteroids, and comet nuclei. The details of the selected spectra are given below. 


\subsection{Visible spectral range}

In the visible range we combined the following spectra: (i) ACOs, (20998) Fountainhills, $H=11.0$, and (44566) 1999 $\mathrm{CK}_{103}, H=11.0$; (ii) D-type asteroids, (3248) Farinella, $H=$ 10.7, and (4744) $1988 \mathrm{RF}_{5}, H=11.1$; (iii) Hilda asteroids, (1529) Oterma, $H=10.05$, and (23186) $2000 \mathrm{PO}_{8}, H=$ 11.1; (iv) Trojan asteroids, (2363) Cebriones, $H=9.11$, and (3709) Polypoites, $H=9.0$; and (v) Cybele asteroids, (2266) Tchaikovsky, $H=10.80$, and (3015) Candy, $H=11.1^{3}$.

Each spectrum was re-binned into 100 bins, fitted to a straight line and normalized by dividing the spectrum by the fit. Finally, the mean spectrum and the standard deviation of each group was computed over each bin, covering a spectral range from $0.55-0.9 \mu \mathrm{m}$.

To quantify the comparison we computed the square difference between the ACO and comparison (COM) reflectances

$R_{\mathrm{r}}=\left(\text { reflectance }_{\mathrm{ACO}}-\text { reflectance }_{\mathrm{COM}}\right)^{2}$

and compared it with the quadratic sum of the standard deviations

$\sigma_{\text {limit }}=2 \sqrt{R_{\mathrm{r}}\left(\sigma_{\mathrm{ACO}}^{2}+\sigma_{\mathrm{COM}}^{2}\right)}$.

If $R_{\mathrm{r}}>3 \times \sigma_{\text {limit }}$ then the differences between the spectra could be significant. No significant differences were found by comparing the spectra, no subtle features appear outside the range of the error associated with each mean spectrum (see Fig. 4).

\subsection{Near infrared spectral range}

We performed the same analysis as seen for the visible spectra with the NIR data. The spectra used in the comparison are: (i) ACOs, (6144) $1994 \mathrm{EQ}_{3}, H=11.5$, (19748) $2000 \mathrm{BD}_{5}$, $H=11.5$, and (20898) Fountainhills; (ii) D-type asteroids, (2569) Madeline, $H=11.2$, and (4744) $1988 \mathrm{RF}_{5}$; and (iii) Hilda asteroids, (2246) Bowell, $H=10.56$, and (23186) $2000 \mathrm{PO}_{8}$. As the sampling in the NIR spectra is quite different from that of the visible spectra, we re-binned the spectra into 50 bins, covering a wavelength range from $0.80-2.35 \mu \mathrm{m}$. Again no subtle features appear in the near infrared.

In view of the results we conclude that no reliable difference is found between the ACO and the comparisons at these wavelengths.

\subsection{Comparison with spectra of comet nuclei}

Recently, high S/N spectra of two comet nuclei were published. Campins et al. (2006) published the visible and near-infrared spectrum of comet 162P/Siding-Spring and Abell et al. (2005) the near-infrared spectrum of $\mathrm{C} / 2001 \mathrm{OG}_{108}$. Even if these comet nuclei are smaller ( $H=13.9$ and 13.0 respectively) than the ACOs considered in Sects. 4.1 and 4.2, we made the same comparison. Results are shown in Fig. 6. The near infrared range of both comet spectra is shorter $(0.8-2.0 \mu \mathrm{m})$ than the range of our ACOs spectra $(0.80-2.35 \mu \mathrm{m})$ because both comets presented significant thermal emission at larger wavelengths. We conclude that in the $0.50-2.0 \mu \mathrm{m}$ there is no subtle feature that can be used to discriminate the cometary or asteroidal nature of the ACOs.

\footnotetext{
3 The Trojan and Cybele spectra are from the available surveys, except Cebriones's spectrum.
}
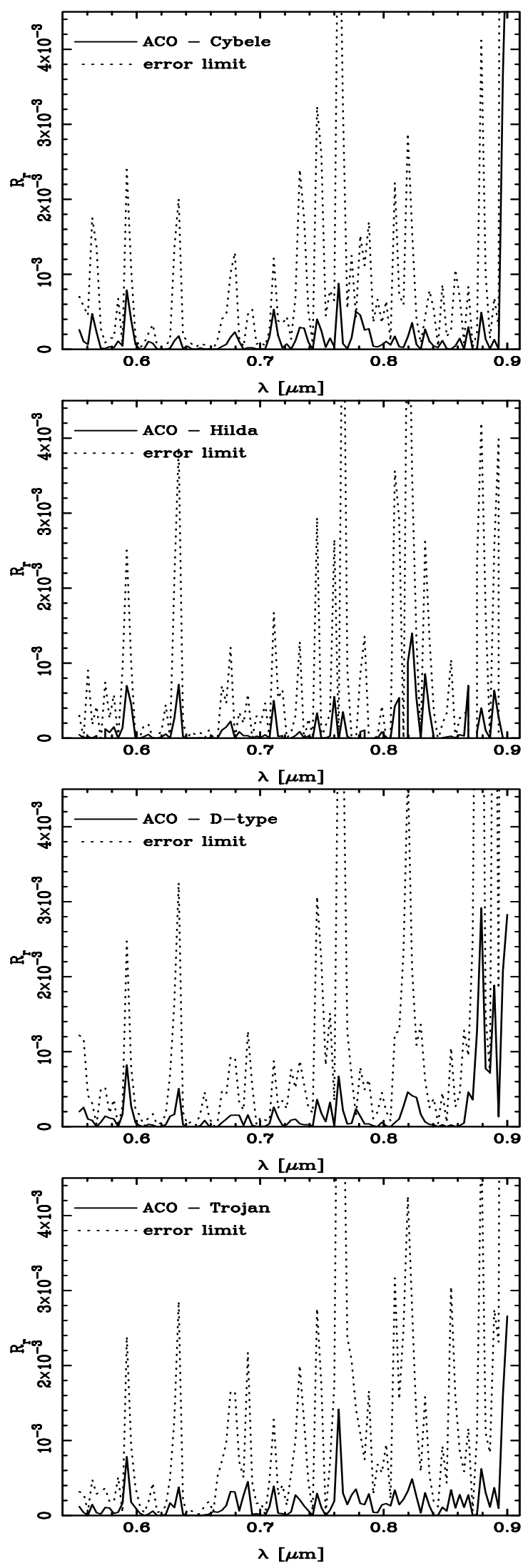

Fig. 4. Comparison of mean spectra between ACO and other populations (see text for details of the calculations). The continuous line shows $R_{\mathrm{r}}$ while the dotted line shows $3 \times \sigma_{\text {limit }}$. No relevant differences were found between them. 

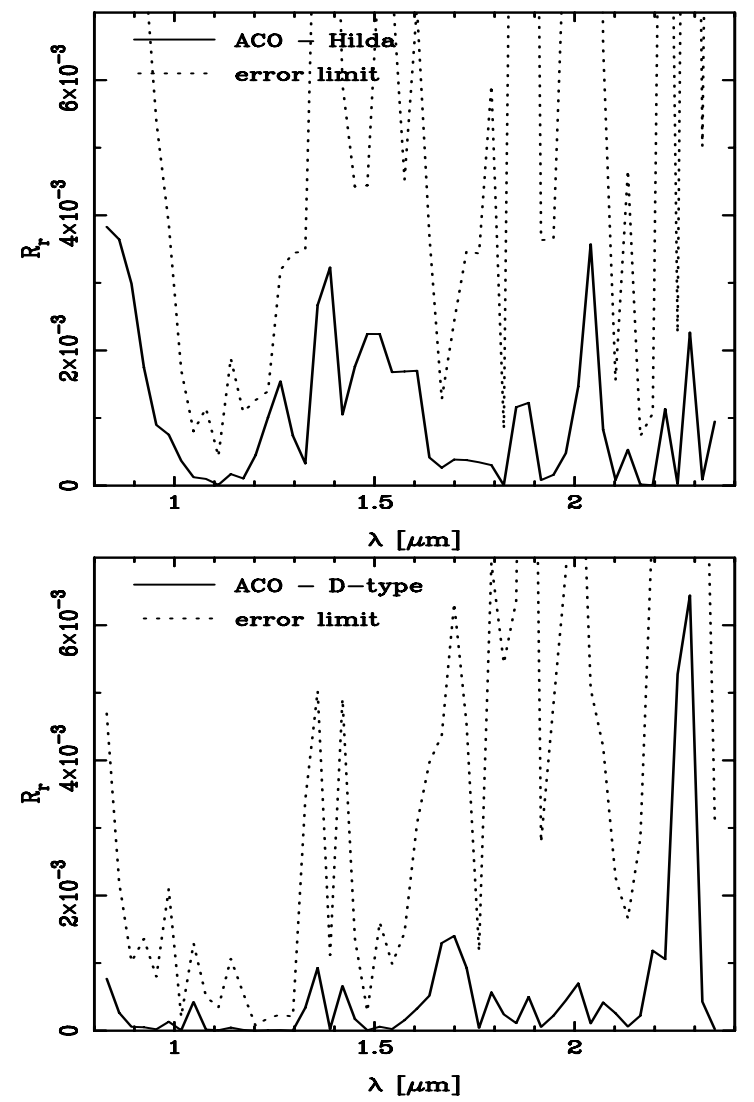

Fig. 5. Same as Fig. 4, but in the NIR range. No relevant differences were found.

\subsection{Individual objects}

Finally, we analyzed the visible and NIR spectrum of every featureless ACO by subtracting the continuum as explained above. Any existent spectral feature should appear as a departure from a horizontal line. Only in the case of (6909) Levison we detect such a feature within the signal-to-noise, its NIR spectrum (Fig. 7) presents a drop at wavelengths larger than $2 \mu \mathrm{m}$. Better data and modeling is needed to confirm this result and to obtain information on the surface components that are producing this absorption.

\section{Featureless ACOs: spectral slope and orbital parameters}

In this section we perform a quantitative analysis in order to compare the featureless ACOs with the other populations dominated by featureless spectra (outer belt and Trojan asteroids, and comets). To do this, the spectra of 18 Trojan and 33 Cybele asteroids were selected from the S3OS2 and SMASS surveys. The total sample, hereafter called the extended sample, has $95 \mathrm{ob}-$ jects and includes 34 ACOs, 19 Trojan, 33 Cybeles, 5 Hilda and 4 D-type inner main-belt asteroids.

The distribution in the space of orbital elements of the $95 \mathrm{ob}-$ jects is shown in Fig. 8, where different symbols indicate different samples. The semi-major axis - eccentricity plot also shows the $T=3$ line (dashed), for inclination equal zero and the $q=1.3 \mathrm{AU}$ line (continuous), which separates the NEO space.
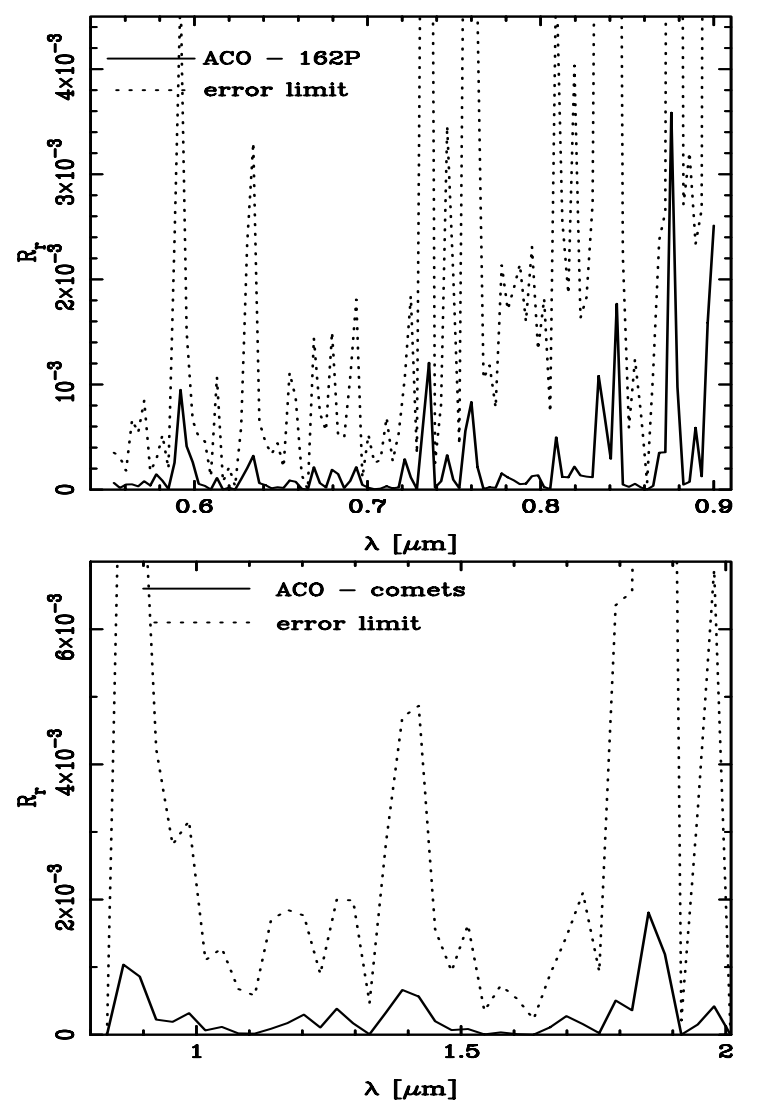

Fig. 6. Comparison of mean spectra between ACO and comet nuclei in the visible (upper plot) and near-infrared (lower plot). The continuous line shows $R_{\mathrm{r}}$ while the dotted line shows $3 \times \sigma_{\text {limit }}$. No relevant differences were found between ACOs and comet spectra.

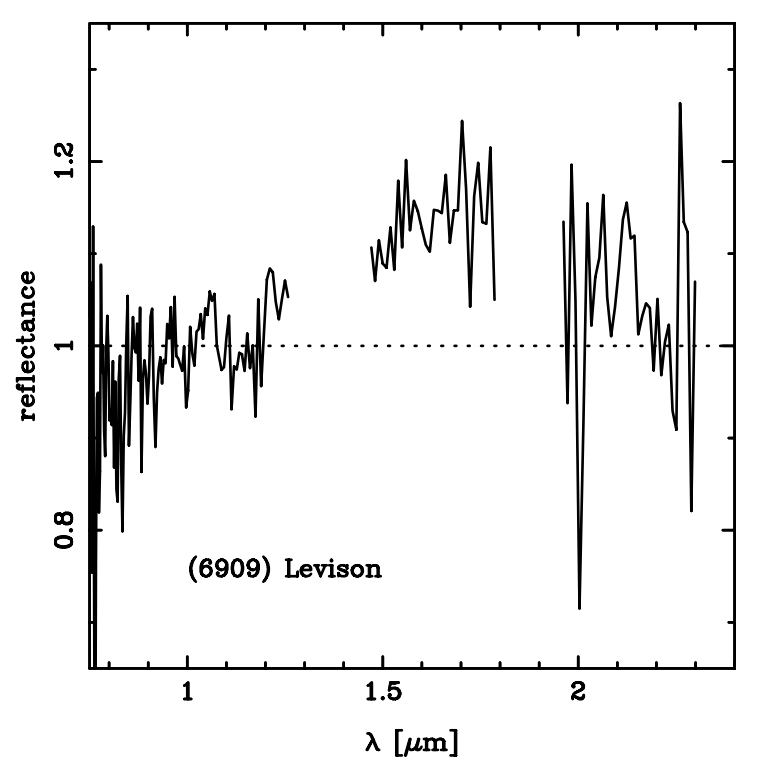

Fig. 7. NIR spectrum of (6909) Levison, notice the downturn in the reflectivity for $\lambda>2.0 \mu \mathrm{m}$.

\subsection{The spectral slope}

Luu \& Jewitt (1996) defined the spectral slope parameter $S^{\prime}$ as

$S^{\prime}=\frac{\Delta S}{\Delta \lambda} \times S^{-1}\left(\lambda_{\text {nor }}\right)$ 


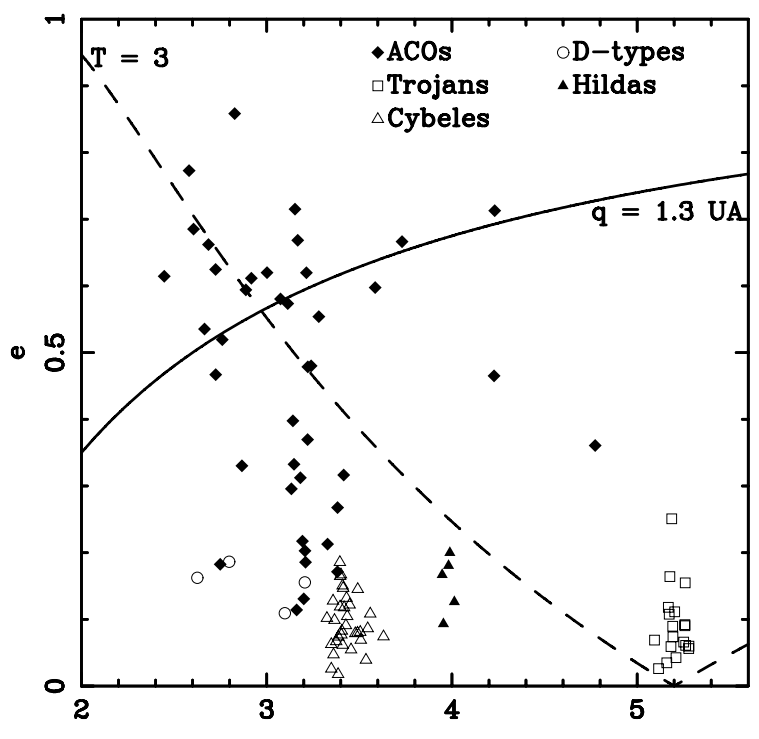

a $[\mathrm{AU}]$

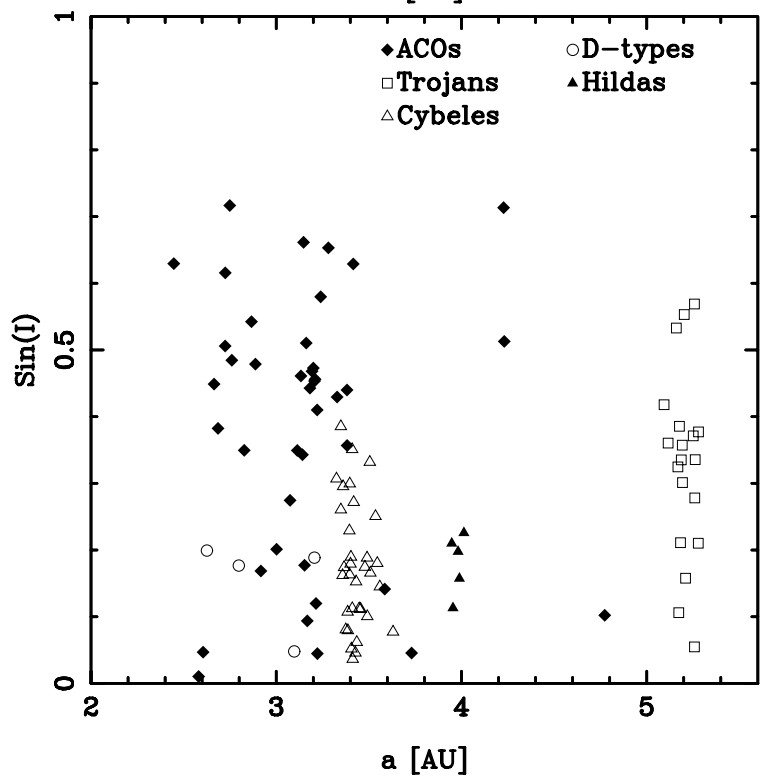

Fig. 8. Orbital elements of the analyzed sample. Upper plot: semi-major axis vs. eccentricity. The continuous line indicates $q=1.3 \mathrm{AU}$, objects above it are NEOs. The dashed line indicates $T=3$, projected onto the $I=0^{\circ}$ plane. Lower plot: semi-major axis vs. $\sin I$. ACOs are indicated by filled rhombi, while Trojan, Hilda, Cybele and D-type main-belt asteroids are indicated by open squares, filled triangles, open triangles and open circles respectively.

where $S\left(\lambda_{\text {nor }}\right)$ is the spectral reflectance of the object at wavelength $\lambda_{\text {nor }} \cdot S^{\prime}$ is a measurement of the variation of $S(\lambda)$ over an interval $\Delta \lambda . S^{\prime}$ is measured in units of $\% / 0.1 \mu \mathrm{m}$.

Assuming that a featureless spectrum has an overall linear shape (as a first order approach), the normalized reflectance can be characterized by a function

$S(\lambda)=S^{\prime} \times \lambda+a$,

where the spectral slope $S^{\prime}$ and $a$ can be computed by a linear fitting of the spectrum.

To consider all objects in our sample, including those that have a spectrum in only one of the spectral ranges, we analyze visible and near-infrared spectra separately. To compute the spectral slopes in both spectral ranges $\left(S_{V}^{\prime}\right.$ and $S_{N}^{\prime}$ in the visible and near-infrared respectively) we first re-binned the visible and the near-infrared spectra into 50 bins. In order to have a uniform spectral coverage, we consider the $0.55-0.90 \mu \mathrm{m}$ spectral range in the visible and the $0.8-2.35 \mu \mathrm{m}$ spectral range in the near-infrared. We make a linear fitting of the visible spectra normalized at $\lambda_{\text {nor }}=0.6 \mu \mathrm{m}$ obtaining the spectral slope in the visible $\left(S_{V}^{\prime}\right)$. We also make a linear fitting of the near-infrared spectra normalized at $\lambda_{\text {nor }}=1.0 \mu \mathrm{m}$.

Notice that a direct comparison between $S_{V}^{\prime}$ and the so obtained spectral slope in the near-infrared is not straightforward as visible and near-infrared spectra are normalized at different wavelengths. So, to compute $S_{N}^{\prime}$ we re-normalize the linear fitting of the near-infrared spectrum at $\lambda_{\text {nor }}=0.6 \mu \mathrm{m}$. Notice that if the spectrum in the $0.5-2.4 \mu \mathrm{m}$ spectral range is a perfect straight line, $S_{N}^{\prime}=S_{V}^{\prime}$ and now a comparison between these two spectral slopes is simple.

The fitted parameters for all the objects of the sample are shown in Table 3, along with their orbital elements ${ }^{4}$ and their absolute magnitude $(H)$ and $T$ values.

\subsection{Search for correlations}

We searched for the existence of correlations between the parameters listed in Table 3 of four different samples of objects. The first includes only 34 ACOs, the second 19 Trojan asteroids, and the third 33 Cybele asteroids. The last sample contains 95 objects of the extended sample as defined above.

The procedure to search for correlations was the following: first, search for correlations among pairs of variables for the ACO sample, then, perform the same search for the other samples. If some correlation appears in the ACO sample and it is not present in other samples, then possibly it is intrinsic to the ACOs. Otherwise, if the correlation also appears in one or more of the other samples it cannot be considered as an intrinsic property of the ACOs.

As noted in Sect. 3.1 and shown by Alvarez-Candal \& Licandro (2006), the NEO and non-NEO sub-populations of the ACOs present different global properties, therefore, we will also analyze these two sub-samples separately. Note that, since none of the comparison samples contain orbits with $q<1.3 \mathrm{AU}$, i.e. NEO orbits, in what follows the acronyms NEO and non-NEO refer only to ACOs.

The Spearman Rank-Order Correlation (Press et al. 1992) was used to test for possible correlations. This test gives two parameters that measure how correlated is a pair of variables. The degree of correlation is measured by $r_{\mathrm{s}}$, where the values 1 , -1 and 0 means total correlation, total anti-correlation and no correlation respectively. The reliability of this parameter is measured by $P_{r_{\mathrm{s}}}$ and the most confident result given by $r_{\mathrm{s}}$ is obtained when $P_{r_{\mathrm{s}}}$ is closer to zero. It is worth mentioning that $\left|r_{\mathrm{s}}\right|>0.5$ denotes a good correlation of the data, if $P_{r_{\mathrm{s}}}$ remains small.

The most interesting results from the applied correlation test are shown in Table 4. In the following we will discuss the possible meaning of these results, with special attention to those relevant to the ACO population. Note that in the first row in Table 4 we included the pair $S_{V}^{\prime}$ vs. phase angle, and $r_{\mathrm{s}} \sim 0$ shows that there is no correlation between them. This indicates that our data are not affected by systematic effects due to phase reddening.

$S_{V}^{\prime}$ vs. $S_{N}^{\prime}$ : as expected, there is a strong correlation between the visible and NIR spectral gradients (see Table 4). This result appears quite evident in Fig. 9 where the $S_{V}^{\prime}$ vs. $S_{N}^{\prime}$ values

${ }^{4} \mathrm{ftp}: / /$ ftp.lowell.edu/pub/elgb/astorb.html 
Table 3. Spectral slopes, orbital parameters and absolute magnitude of the sample of featureless ACOs, and the D-type inner main-belt, Hilda, Trojan and Cybele asteroids. $I$ is the inclination respect to the orbit of Jupiter.

\begin{tabular}{|c|c|c|c|c|c|c|c|c|}
\hline Object & $S_{V}^{\prime}$ & $S_{N}^{\prime}$ & $\overline{\left.I I{ }^{0}\right]}$ & $e$ & $a[\mathrm{AU}]$ & $\overline{\mathrm{H}}$ & $\bar{T}$ & Class. \\
\hline (65) Cybele & $0.95 \ddagger$ & $-\frac{N}{-}$ & 3.548 & 0.1046 & 3.4348 & 6.62 & 3.106 & Cybele \\
\hline (87) Sylvia & $4.14 \ddagger$ & - & 10.856 & 0.0796 & 3.4904 & 6.94 & 3.078 & Cybele \\
\hline (107) Camilla & $2.04 \div$ & - & 10.048 & 0.0789 & 3.4785 & 7.08 & 3.084 & Cybele \\
\hline (168) Sibylla & $0.95 \ddagger$ & - & 4.634 & 0.0669 & 3.3753 & 7.94 & 3.121 & Cybele \\
\hline (225) Henrietta & -2.43 & - & 20.903 & 0.2677 & 3.3829 & 8.72 & 3.001 & ACO \\
\hline (229) Adelinda & $0.74 \div$ & - & 2.092 & 0.1469 & 3.4130 & 9.13 & 3.102 & Cybele \\
\hline (260) Huberta & $3.16 \neq$ & - & 6.442 & 0.1219 & 3.4475 & 8.97 & 3.095 & Cybele \\
\hline (414) Liriope & $0.96 \%$ & - & 9.542 & 0.0691 & 3.5081 & 9.49 & 3.082 & Cybele \\
\hline (522) Helga & $3.09 \pm$ & - & 4.436 & 0.0742 & 3.6306 & 9.12 & 3.076 & Cybele \\
\hline (536) Merapi & $3.05 \%$ & - & 19.380 & 0.0809 & 3.5053 & 8.08 & 3.016 & Cybele \\
\hline (713) Luscinia & $1.25 \ddagger$ & - & 10.327 & 0.1672 & 3.4001 & 8.97 & 3.079 & Cybele \\
\hline (721) Tabora & $6.89 \div$ & - & 8.344 & 0.1085 & 3.5593 & 9.26 & 3.072 & Cybele \\
\hline (790) Pretoria & $3.95 \ddagger$ & - & 20.549 & 0.1505 & 3.4098 & 8.00 & 3.011 & Cybele \\
\hline (911) Agamemnon & $6.76 \%$ & - & 21.791 & 0.0659 & 5.2513 & 7.80 & 2.868 & Trojan \\
\hline (1004) Belopolskya & $4.66 \ddagger$ & - & 2.977 & 0.0827 & 3.4058 & 9.99 & 3.115 & Cybele \\
\hline (1028) Lydina & $1.17 \ddagger$ & - & 9.391 & 0.1189 & 3.3964 & 9.43 & 3.095 & Cybele \\
\hline (1143) Odysseus & $12.56 \div$ & - & 3.135 & 0.0904 & 5.2576 & 7.93 & 2.992 & Trojan \\
\hline (1154) Astronomia & $3.72 \ddagger$ & - & 4.559 & 0.0739 & 3.3868 & 10.51 & 3.118 & Cybele \\
\hline (1177) Gonnessia & $0.87 \ddagger$ & - & 15.103 & 0.0259 & 3.3489 & 9.30 & 3.085 & Cybele \\
\hline (1266) Tone & $6.55 \ddagger$ & - & 17.180 & 0.0472 & 3.3604 & 9.41 & 3.066 & Cybele \\
\hline (1280) Baillauda & $4.33 \ddagger$ & - & 6.458 & 0.0615 & 3.4102 & 10.33 & 3.111 & Cybele \\
\hline (1328) Devota & $14.22 \ddagger$ & - & 5.770 & 0.1453 & 3.4916 & 10.31 & 3.083 & Cybele \\
\hline (1345) Potomac & 3.62 & 3.49 & 11.399 & 0.1806 & 3.9817 & 9.73 & 2.993 & Hilda \\
\hline (1362) Griqua & $-0.74 \ddagger$ & 0.68 & 24.206 & 0.3696 & 3.2207 & 11.18 & 2.962 & $\mathrm{ACO}$ \\
\hline (1373) Cincinnati & $3.52 \ddagger$ & - & 38.968 & 0.3164 & 3.4157 & 11.20 & 2.740 & $\mathrm{ACO}$ \\
\hline (1529) Oterma & 8.61 & 7.67 & 9.054 & 0.1999 & 3.9889 & 10.05 & 2.999 & Hilda \\
\hline (1556) Wingolfia & $5.73 \ddagger$ & - & 15.770 & 0.1178 & 3.4181 & 10.55 & 3.056 & Cybele \\
\hline (1574) Meyer & $10.66 \ddagger$ & - & 14.512 & 0.0391 & 3.5362 & 10.30 & 3.053 & Cybele \\
\hline (1579) Herrick & $-1.24 \ddagger$ & - & 8.780 & 0.1319 & 3.4309 & 10.68 & 3.088 & Cybele \\
\hline (1542) Schalen & 10.51 & 9.17 & 2.763 & 0.1090 & 3.0973 & 10.30 & 3.211 & D-Type \\
\hline (1749) Telamon & $10.79 \ddagger$ & - & 6.088 & 0.1077 & 5.1732 & 9.20 & 2.982 & Trojan \\
\hline (1754) Cunningham & 4.31 & 2.92 & 12.121 & 0.1669 & 3.9473 & 9.77 & 2.997 & Hilda \\
\hline (1796) Riga & $2.03 \ddagger$ & - & 22.668 & 0.0626 & 3.3488 & 9.84 & 3.017 & Cybele \\
\hline (1841) Masaryk & $3.06 \div$ & - & 2.632 & 0.0910 & 3.4276 & 10.80 & 3.110 & Cybele \\
\hline (1922) Zulu & - & 5.59 & 35.429 & 0.4801 & 3.2397 & 12.20 & 2.752 & $\mathrm{ACO}$ \\
\hline (2246) Bowell & - & 6.40 & 6.496 & 0.0932 & 3.9542 & 10.56 & 3.040 & Hilda \\
\hline (2266) Tchaikovsky & $9.65 \ddagger$ & - & 13.245 & 0.1856 & 3.3951 & 10.80 & 3.060 & Cybele \\
\hline (2363) Cebriones & 12.43 & 5.52 & 32.197 & 0.0348 & 5.1599 & 9.11 & 2.693 & Trojan \\
\hline (2569) Madeline & - & 11.14 & 11.477 & 0.1624 & 2.6261 & 11.20 & 3.147 & D-Type \\
\hline (2634) James Bradley & $3.00 \%$ & - & 6.402 & 0.0546 & 3.4552 & 10.20 & 3.103 & Cybele \\
\hline (2891) McGetchin & $9.83 \div$ & - & 9.333 & 0.1277 & 3.3582 & 11.20 & 3.101 & Cybele \\
\hline (2906) Caltech & $1.82 \ddagger$ & - & 30.690 & 0.1141 & 3.1616 & 10.00 & 2.995 & ACO \\
\hline (2920) Automedon & $9.24 \div$ & - & 21.117 & 0.0262 & 5.1156 & 8.80 & 2.880 & Trojan \\
\hline (2938) Hopi & 1.51 & 2.80 & 41.409 & 0.3325 & 3.1473 & 11.50 & 2.775 & $\mathrm{ACO}$ \\
\hline (3015) Candy & $7.26 \%$ & - & 17.426 & 0.1646 & 3.3965 & 11.10 & 3.037 & Cybele \\
\hline (3063) Makhaon & $8.49 \div$ & - & 12.178 & 0.0592 & 5.1825 & 8.60 & 2.961 & Trojan \\
\hline (3141) Buchar & $10.19 \ddagger$ & - & 10.932 & 0.0769 & 3.4035 & 10.50 & 3.094 & Cybele \\
\hline (3248) Farinella & 11.10 & 6.05 & 10.868 & 0.1553 & 3.2066 & 10.70 & 3.145 & D-Type \\
\hline (3451) Mentor & $1.45 \dagger$ & - & 24.701 & 0.0689 & 5.0935 & 8.10 & 2.830 & Trojan \\
\hline (3552) Don Quixote & $16.22 \dagger$ & - & 30.853 & 0.7128 & 4.2311 & 13.00 & 2.330 & $\mathrm{ACO}$ \\
\hline (3688) Navajo & 2.66 & 1.90 & 2.560 & 0.4786 & 3.2219 & 14.90 & 2.996 & $\mathrm{ACO}$ \\
\hline (3709) Polypoites & $11.64 \ddagger$ & - & 19.604 & 0.0611 & 5.2616 & 9.00 & 2.895 & Trojan \\
\hline (3793) Leonteus & $7.39 \ddagger$ & - & 20.930 & 0.0897 & 5.1922 & 8.80 & 2.875 & Trojan \\
\hline (4035) 1986 WD & $8.79 \div$ & - & 12.135 & 0.0562 & 5.2782 & 9.30 & 2.962 & Trojan \\
\hline (4060) Deipylos & $0.07 \ddagger$ & - & 16.145 & 0.1548 & 5.2593 & 8.90 & 2.910 & Trojan \\
\hline (4063) Euforbo & $7.81 \%$ & - & 18.949 & 0.1181 & 5.1683 & 8.60 & 2.892 & Trojan \\
\hline (4068) Menestheus & $10.07 \ddagger$ & - & 17.526 & 0.0738 & 5.1930 & 9.40 & 2.915 & Trojan \\
\hline (4489) $1988 \mathrm{AK}$ & $8.63 \ddagger$ & - & 22.157 & 0.0598 & 5.2794 & 9.00 & 2.864 & Trojan \\
\hline (4744) $1988 \mathrm{RF}_{5}$ & 13.19 & 8.50 & 10.177 & 0.1864 & 2.7976 & 11.10 & 3.034 & D-Type \\
\hline (4833) Meges & $11.67 \ddagger$ & - & 34.672 & 0.0921 & 5.2579 & 9.10 & 2.657 & Trojan \\
\hline (4835) $1989 \mathrm{BQ}$ & $8.35 \ddagger$ & - & 19.582 & 0.2509 & 5.1867 & 9.80 & 2.837 & Trojan \\
\hline (4837) Bickerton & -1.01 & - & 28.223 & 0.1307 & 3.2004 & 11.60 & 3.012 & $\mathrm{ACO}$ \\
\hline (4902) Thessandrus & $8.14 \div$ & - & 9.071 & 0.0426 & 5.2095 & 9.60 & 2.981 & Trojan \\
\hline (4997) Ksana & $-1.37 \ddagger$ & - & 32.852 & 0.3303 & 2.8662 & 11.90 & 3.001 & $\mathrm{ACO}$ \\
\hline
\end{tabular}


Table 3. continued.

\begin{tabular}{|c|c|c|c|c|c|c|c|c|}
\hline Object & $\overline{S_{V}^{\prime}}$ & $\overline{S_{N}^{\prime}}$ & $\overline{I I\left[{ }^{\circ}\right]}$ & $\bar{e}$ & $a$ a [AU] & $\overline{\mathrm{H}}$ & $\bar{T}$ & Class. \\
\hline (5264) Telephus & 10.21 末 & - & 33.583 & 0.1113 & 5.2025 & 9.50 & 2.676 & Trojan \\
\hline (5301) Novobranets & $1.11 \ddagger$ & - & 10.024 & 0.0989 & 3.3658 & 11.30 & 3.102 & Cybele \\
\hline (5362) $1978 \mathrm{CH}$ & $7.15 \ddagger$ & - & 6.153 & 0.0177 & 3.3853 & 11.50 & 3.119 & Cybele \\
\hline (5648) $1990 \mathrm{VU}_{1}$ & $13.46 \%$ & - & 22.674 & 0.1642 & 5.1766 & 9.20 & 2.835 & Trojan \\
\hline (5914) Kathywhaler & $8.74 \%$ & - & 10.385 & 0.0863 & 3.5455 & 10.80 & 3.069 & Cybele \\
\hline (6057) Robbia & $2.35 \ddagger$ & - & 17.872 & 0.1018 & 3.3250 & 11.10 & 3.062 & Cybele \\
\hline (6144) $1994 \mathrm{EQ}_{3}$ & 7.54 & 6.11 & 5.864 & 0.3607 & 4.7736 & 11.50 & 2.871 & $\mathrm{ACO}$ \\
\hline (6909) Levison & 1.98 & 0.96 & 37.995 & 0.4669 & 2.7246 & 14.10 & 2.935 & $\mathrm{ACO}$ \\
\hline (7604) Kridsadaporn & -0.87 † & - & 20.448 & 0.5736 & 3.1136 & 13.70 & 2.868 & $\mathrm{ACO}$ \\
\hline (8373) Stephengould & 14.84 & 2.92 & 40.782 & 0.5540 & 3.2816 & 13.80 & 2.606 & $\mathrm{ACO}$ \\
\hline (10739) Lowman & 2.17 & - & 20.056 & 0.3977 & 3.1416 & 14.50 & 3.006 & $\mathrm{ACO}$ \\
\hline (14409) $1991 \mathrm{RM}_{1}$ & 1.17 & 3.01 & 27.915 & 0.2173 & 3.1932 & 11.60 & 2.996 & $\mathrm{ACO}$ \\
\hline (18898) $2000 \mathrm{JX}$ & 5.26 & 2.74 & 26.283 & 0.3123 & 3.1819 & 12.50 & 2.982 & $\mathrm{ACO}$ \\
\hline (19748) $2000 \mathrm{BD}_{5}$ & 5.20 & 3.37 & 27.131 & 0.1857 & 3.2098 & 11.50 & 3.010 & $\mathrm{ACO}$ \\
\hline (20898) Fountainhills & 12.69 & 4.63 & 45.512 & 0.4651 & 4.2275 & 11.00 & 2.374 & $\mathrm{ACO}$ \\
\hline (22653) $1998 \mathrm{QW}_{2}$ & - & 0.03 & 45.776 & 0.1826 & 2.7488 & 13.60 & 2.912 & $\mathrm{ACO}$ \\
\hline (22714) $1998 \mathrm{SR}_{2}$ & 4.54 & 2.37 & 26.965 & 0.2028 & 3.2069 & 12.60 & 3.008 & $\mathrm{ACO}$ \\
\hline (23186) $2000 \mathrm{PO}_{8}$ & 10.80 & 6.98 & 13.049 & 0.1263 & 4.0130 & 11.10 & 2.994 & Hilda \\
\hline (24689) $1990 \mathrm{OH}_{1}$ & -0.01 & - & 27.456 & 0.2958 & 3.1332 & 12.80 & 2.999 & $\mathrm{ACO}$ \\
\hline (44566) $1999 \mathrm{CK}_{103}$ & 5.19 & 3.36 & 25.430 & 0.2128 & 3.3288 & 11.60 & 2.989 & $\mathrm{ACO}$ \\
\hline (65996) $1998 \mathrm{MX}_{5}$ & 2.61 & 2.34 & 9.706 & 0.6115 & 2.9171 & 18.50 & 2.955 & $\mathrm{ACO}$ \\
\hline (85490) $1997 \mathrm{SE}_{5}$ & $6.83 \dagger$ & - & 2.609 & 0.6664 & 3.7307 & 14.80 & 2.657 & $\mathrm{ACO}$ \\
\hline (102528) $1999 \mathrm{US}_{3}$ & 3.52 & 3.24 & 28.987 & 0.5194 & 2.7604 & 13.70 & 2.986 & $\mathrm{ACO}$ \\
\hline 1992 UB & $6.19 \dagger$ & - & 15.931 & 0.5804 & 3.0749 & 15.98 & 2.903 & $\mathrm{ACO}$ \\
\hline $1999 \mathrm{SE}_{10}$ & $2.37 \dagger$ & - & 6.888 & 0.6198 & 3.2146 & 20.04 & 2.846 & $\mathrm{ACO}$ \\
\hline $2000 \mathrm{PG}_{3}$ & $11.37 \dagger$ & - & 20.455 & 0.8584 & 2.8272 & 15.81 & 2.554 & $\mathrm{ACO}$ \\
\hline $2000 \mathrm{WL}_{10}$ & $2.29 \dagger$ & - & 10.204 & 0.7152 & 3.1529 & 17.99 & 2.725 & $\mathrm{ACO}$ \\
\hline $2001 \mathrm{TX}_{16}$ & $3.91 \dagger$ & - & 8.135 & 0.5975 & 3.5856 & 13.90 & 2.773 & $\mathrm{ACO}$ \\
\hline $2001 \mathrm{UC}_{5}$ & $3.63 \dagger$ & - & 30.391 & 0.6246 & 2.7243 & 21.32 & 2.897 & $\mathrm{ACO}$ \\
\hline $2001 \mathrm{UU}_{92}$ & $5.57 \dagger$ & - & 5.382 & 0.6684 & 3.1675 & 19.91 & 2.800 & $\mathrm{ACO}$ \\
\hline $2004 \mathrm{JR}_{1}$ & 1.05 & - & 28.609 & 0.5940 & 2.8874 & 17.56 & 2.866 & $\mathrm{ACO}$ \\
\hline
\end{tabular}

$\dagger$ Spectrum from the SMASS.

$\$$ Spectrum from the S3OS2.

Table 4. Results of the Spearman Rank-Order Correlations between $S_{V}^{\prime}$ and the phase angle $\alpha, S_{N}^{\prime}$, the Tisserand parameter $T$, the orbital elements of the asteroids $\left(a, e, i\right.$, and their nuclear mangnitude $H . r_{\mathrm{s}}$ is a measurement of the degree of correlation between the two parameters, $P_{r_{\mathrm{s}}}$ is a measurement of the reliability of $r_{\mathrm{s}}$ (see text).

\begin{tabular}{lcccccccc}
\hline \hline variables & $r_{\mathrm{s}}(\mathrm{ACO})$ & $P_{r_{\mathrm{s}}}(\mathrm{ACO})$ & $r_{\mathrm{s}}($ Tro $)$ & $P_{r_{\mathrm{s}}}($ Tro $)$ & $r_{\mathrm{s}}(\mathrm{Cyb})$ & $P_{r_{\mathrm{s}}}(\mathrm{Cyb})$ & $r_{\mathrm{s}}($ all $)$ & $P_{r_{\mathrm{s}}}($ all $)$ \\
\hline$S_{V}^{\prime}$ vs. $\alpha$ & - & - & - & - & - & - & -0.01 & 0.9656 \\
$S_{V}^{\prime}$ vs. $S_{N}^{\prime}$ & 0.63 & 0.0165 & - & - & - & - & 0.70 & 0.0003 \\
$S_{V}^{\prime}$ vs. $T$ & -0.52 & 0.0019 & -0.06 & 0.7918 & -0.25 & 0.1658 & -0.21 & 0.0405 \\
$S_{V}^{\prime}$ vs. $a$ & 0.36 & 0.0392 & 0.03 & 0.9148 & 0.15 & 0.4109 & 0.42 & $3 \times 10^{-5}$ \\
$S_{V}^{\prime}$ vs. $e$ & 0.37 & 0.0344 & -0.01 & 0.9716 & 0.04 & 0.8419 & -0.14 & 0.1855 \\
$S_{V}^{\prime}$ vs. $\sin I$ & -0.12 & 0.5144 & 0.13 & 0.6064 & 0.17 & 0.3325 & 0.01 & 0.9173 \\
$S_{V}^{\prime}$ vs. H & 0.19 & 0.2875 & 0.29 & 0.2241 & 0.39 & 0.0235 & -0.08 & 0.4371 \\
\hline
\end{tabular}

for the ACOs (left panel), and for the whole sample of featureless objects, 22 in total ${ }^{5}$ (right panel) are plotted. This is similar to the known correlation found in the color-color diagrams for Centaurs and TNOs (e.g. Barucci et al. 2004). Notice that for most objects $S_{V}^{\prime}=S_{N}^{\prime}$ considering the error bars. But while, within the error bars, there are no objects with $S_{V}^{\prime}<S_{N}^{\prime}$, there are several with $S_{V}^{\prime}>S_{N}^{\prime}$, that means, a significant number of objects in the sample are redder in the visible than in the nearinfrared. In fact, most objects are below the $S_{V}^{\prime}=S_{N}^{\prime}$ line in Fig. 9, supporting that most featureless objects are redder in the visible than in the near-infrared. Notice also that, even if it is possible to make a linear fitting to the data and compute, e.g., $S_{V}^{\prime}$ from $S_{N}^{\prime}$ in case we only have the near-infrared spectrum,

\footnotetext{
5 Note that in this case only objects with complete VNIR spectrum are considered.
}

results can be very inaccurate, in particular if the object is very red.

So, in the further analysis we will use only the computed $S_{V}^{\prime}$ to search for correlations with the other parameters, as the number of objects with obtained visible spectrum is larger than those with near-infrared spectrum.

$S_{V}^{\prime}$ vs. Tisserand parameter: a strong anti-correlation appears when considering the ACO sample; having $r_{\mathrm{s}}=-0.52$, $P_{r_{\mathrm{s}}}=0.0019$ (see Fig. 10 and Table 4). On the other hand, the Trojan and the Cybele samples show a weaker anti-correlation with -0.06 and -0.25 , respectively. A weak anti-correlation seems to exist in the whole sample, $r_{\mathrm{s}}=-0.21, P_{r_{\mathrm{s}}}=0.0405$, possibly due to the strong anti-correlation of the ACO population. Therefore, this correlation seems to be intrinsic of the ACOs meaning that the reddest ACOs have the lowest $T$. The trend is also found in both NEO and non-NEO populations 

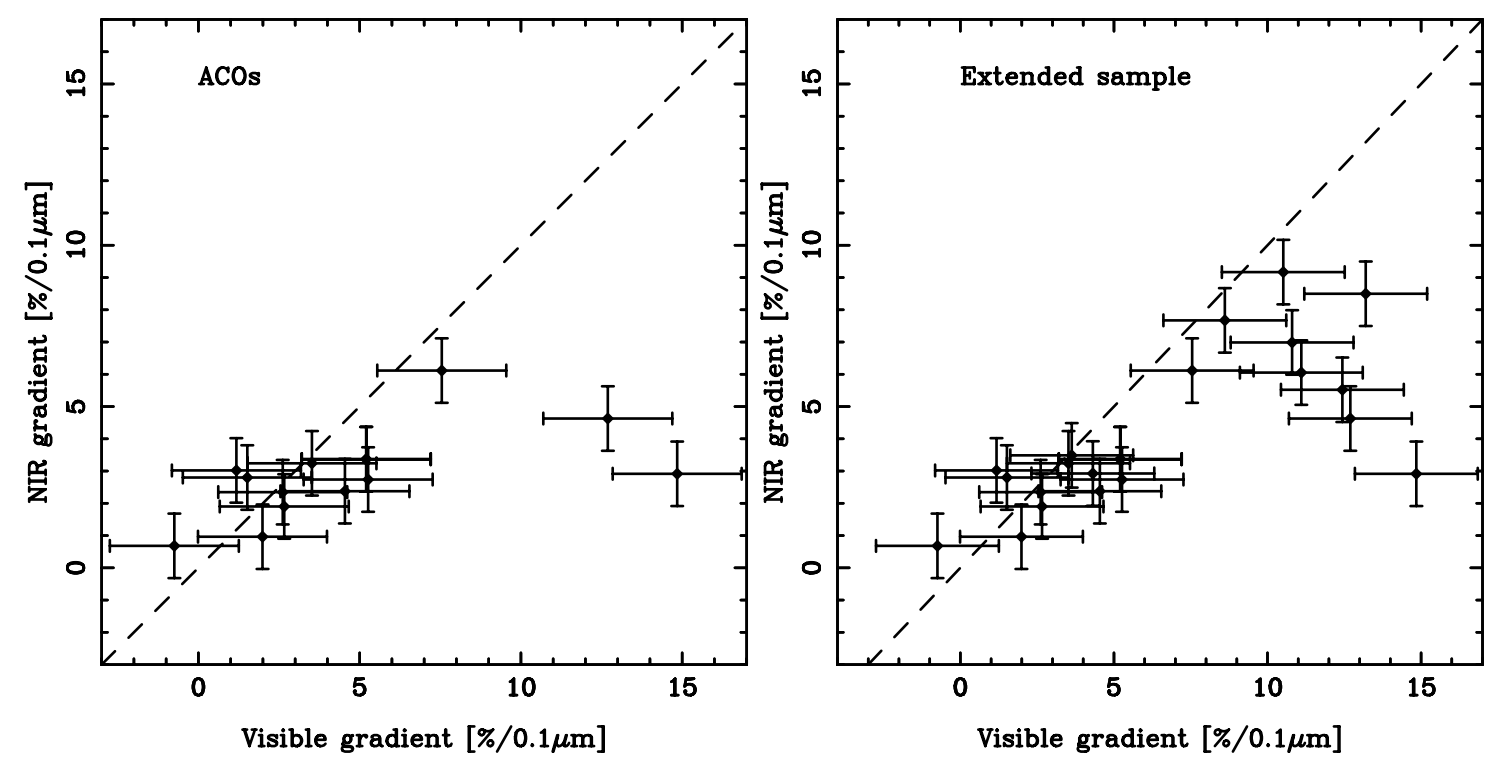

Fig. 9. $S_{V}^{\prime}$ vs. $S_{N}^{\prime}$ parameters plots of the ACOs population (left panel) and the whole extended sample including ACOs, inner main-belt D-types, Cybele, Hilda and Trojan asteroids (right panel). Only objects with visible and near-infrared spectra are included. Notice the (expected) strong correlation between both spectral slopes, and also that most objects have $S_{V}^{\prime}>S_{N}^{\prime}$ and none have $S_{V}^{\prime}<S_{N}^{\prime}$ (considering the error bars).
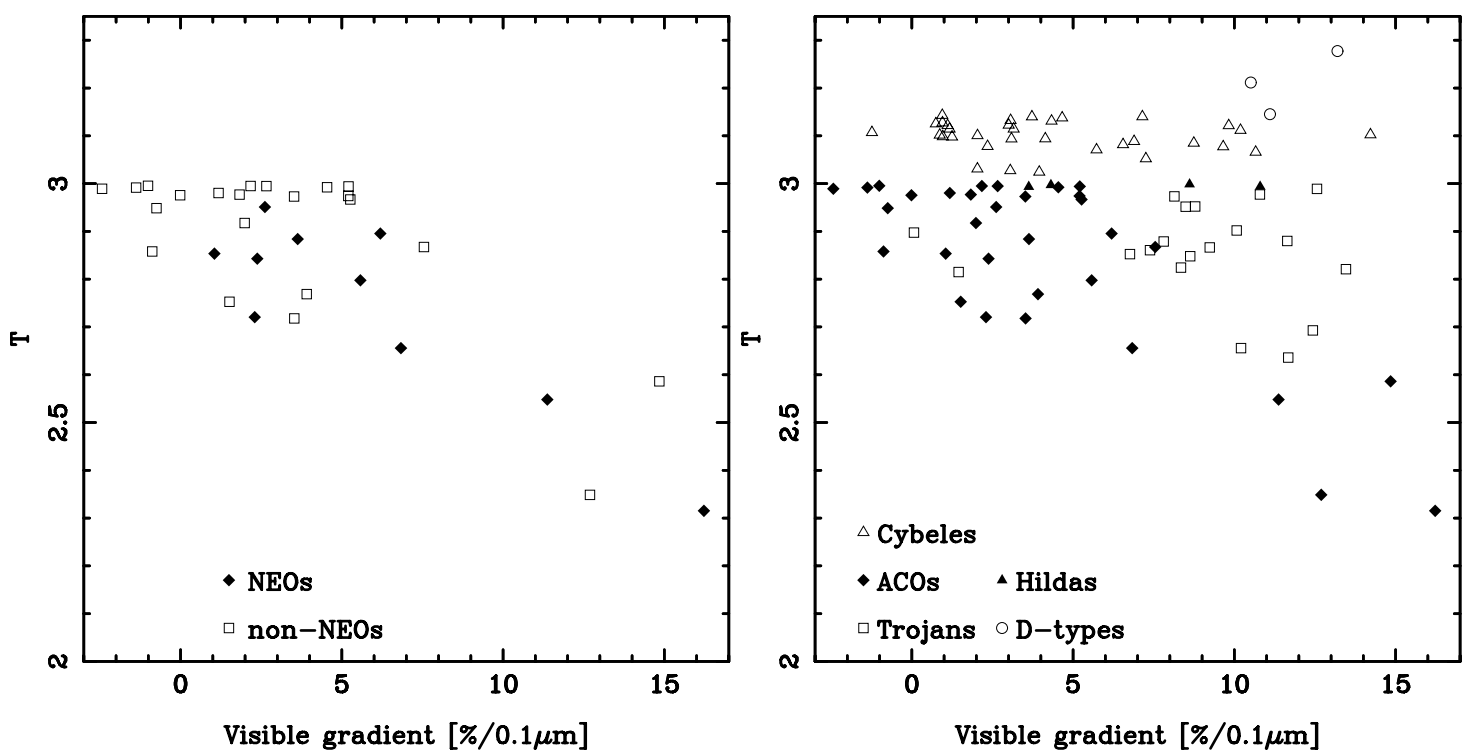

Fig. 10. $S_{V}^{\prime}$ vs. Tisserand parameter plots of the ACOs population (left panel) and the whole extended sample including ACOs, inner main-belt D-types, Cybele, Hilda and Trojan asteroids (right panel). Notice the strong correlation in the ACOs population.

considered separately. It is worth noting that the correlation is strongly influenced by the presence of four very red objects, including (3552) Don Quixote, which was considered by Bottke et al. (2002) as one of the most probable extinct comet nuclei.

An interesting feature is that there is no object, of any of the featureless objects analyzed here, that appears in the blue-neutral space with small $T$. Further dynamical study is needed to better understand this interesting result.

$S_{V}^{\prime}$ vs. semi-major axis: a weak correlation appears between these parameters (see Fig. 11 and Table 4). The correlation seems not to be exclusive of the ACOs since it is also present in most of the other samples with the exception of Trojan asteroids, as all of them have similar semi-major axis. This indicates that objects farthest from the sun are reddest, as was already pointed out by diverse authors (Vilas \& Smith 1985; Hartmann et al. 1987; Lagerkvist et al. 1993; Carvano et al. 2003). The NEO and
non-NEO samples share the overall behavior of the total ACO sample, though with different degrees of reliability.

$S_{V}^{\prime}$ vs. eccentricity: another weak correlation appears between these parameters (see Fig. 12 and Table 4), suggesting that higher eccentricities are associated with redder spectra only in the ACOs population. This should be associated with the degree of dynamical excitation of the orbit (even though there is no correlation at all between inclination and $S_{V}^{\prime}$ ). The comparison samples do not show any correlation due to the presence of very red objects with low eccentricities and the lack of high eccentricity objects.

$S_{V}^{\prime}$ vs. absolute magnitude: for the whole ACO population we did not find any correlation between color $\left(S_{V}^{\prime}\right)$ and absolute magnitude $\left(H_{V}\right.$, see Fig. 13). This is clear in the case of non-NEO ACOs. But the NEOs show an anti-correlation $\left(r_{\mathrm{s}}=-0.6, P_{r_{\mathrm{s}}}=0.05\right)$ which means that the fainter objects 

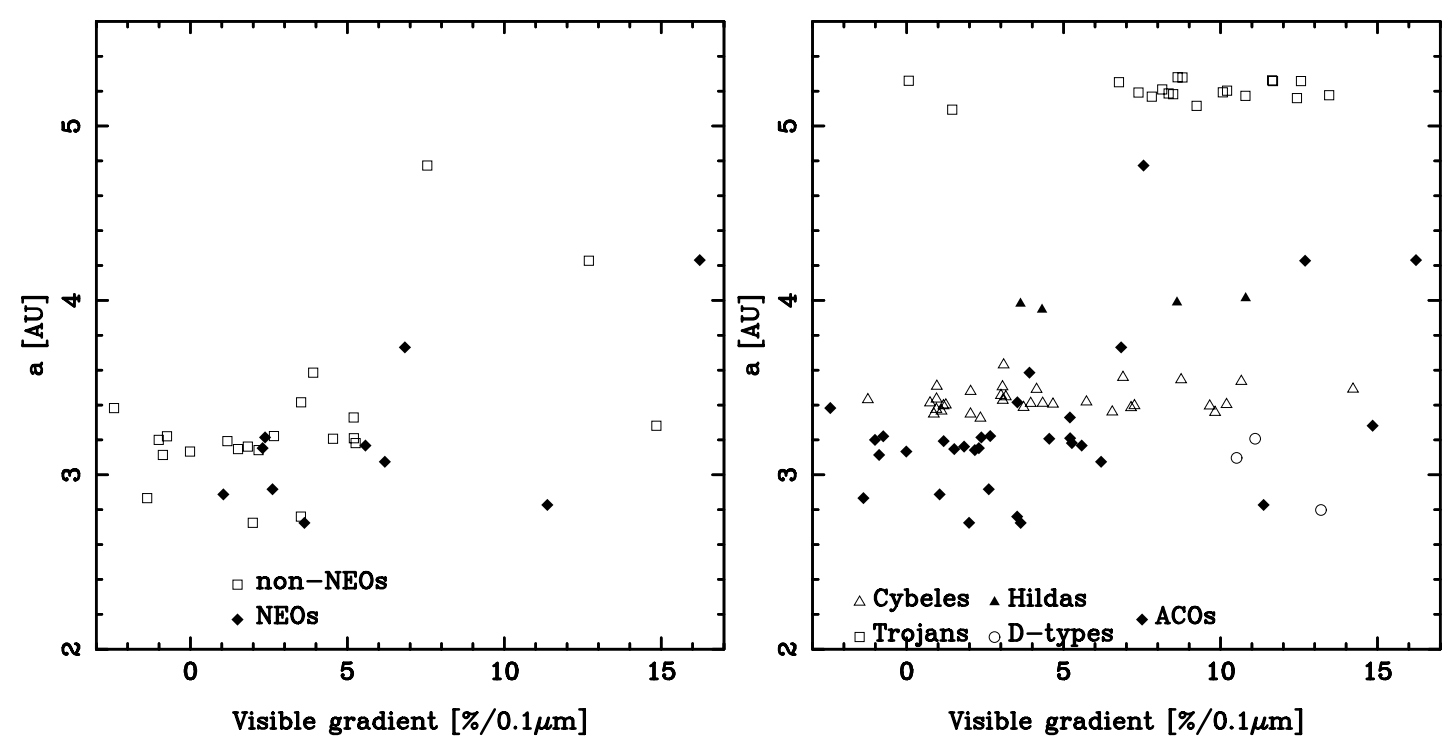

Fig. 11. $S_{V}^{\prime}$ vs. $a$ parameters plots of the ACOs population (left panel) and the whole extended sample (right panel). Notice that there is a weak correlation in all populations (but Trojan asteroids) that suggests that far from the Sun the objects are redder (see text).
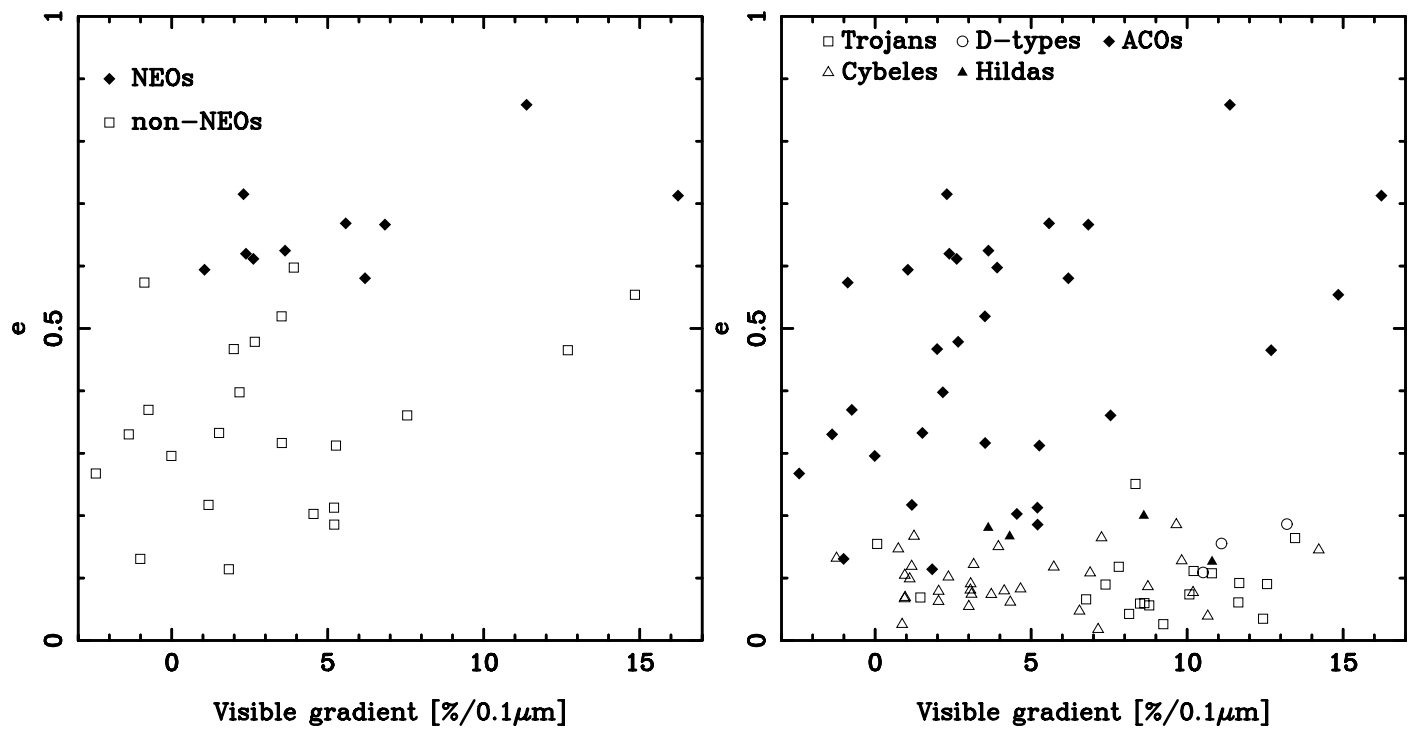

Fig. 12. $S_{V}^{\prime}$ vs. $e$ parameters plots of the ACOs population (left panel) and the whole extended sample (right panel). Notice that there is a weak correlation in the ACOs population. No correlation is found in any of the other samples.

are bluer while the brightest ones are redder. Anyhow we have to mention that this is based on only two red objects in the population, and that the size is introducing a strong bias, as faint (small) objects are only detected in the NEO population. More data are needed to confirm this correlation.

No other correlation appeared (as shown in Table 4). In particular, since we found correlations for the ACOs sample in semi-major axis and eccentricity, we also searched for correlations in the $S_{V}^{\prime}$ vs. perihelion distance pair, but with negative results.

The correlations found seem to relate dynamical and surface properties of ACOs. Particularly striking is the anti-correlation found between $S_{V}^{\prime}$ and $T$, with the reddest ACOs having the lowest Tisserand parameter. It is important to note that this relation is shared by both sub-populations of ACOs, NEOs and non-NEOs.

By comparing these results with results from the dynamical evolution of JFC (Alvarez-Candal \& Roig 2004) we have found that, in the perihelion distance - Tisserand parameter space, hereafter $q-T$ space, most of the ACOs are concentrated in a region where the dynamical lifetime scales of JFC are in the order of $10^{4} \mathrm{yr}$, comparable with the physical lifetime of JFC as suggested by Levison \& Duncan (1997). That region is $(2.7<T \leq 3 ; 0.6<q<1.8 \mathrm{AU}$ ) (see Fig. 3). Comparing with Fig. 10, that range of $T$ corresponds to bluer ACOs, as well as with the majority of the ACOs with bands. Therefore, that region seems to be shared by bona fide (interlopers) asteroids and dormant/dead comets.

On the other hand, the reddest ACOs have $2.7 \leq T$ and they are located in very unstable regions of the $q-T$ space. Therefore they should have been injected onto such orbits recently.

The weak correlation found between $S_{V}^{\prime}$ and eccentricity is related to the anti-correlation found between $T$ and $S_{V}^{\prime}$. This arises from the relationship between $T$ and eccentricity through

$\left(T-\frac{a_{J}}{a}\right)^{2} \propto\left(1-e^{2}\right)$ 

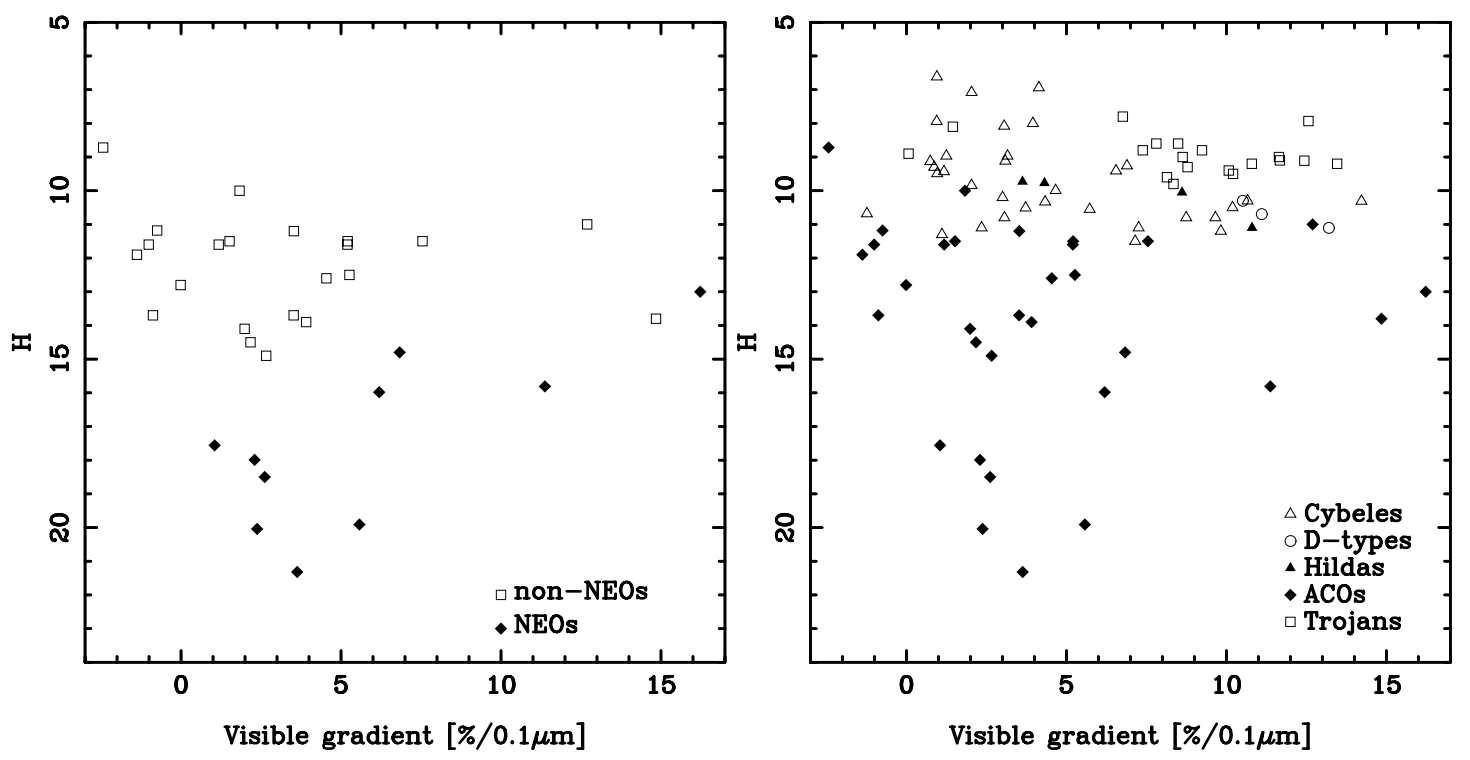

Fig. 13. Color-size ( $S_{V}^{\prime}$ vs. $\left.H_{V}\right)$ plots of the ACOs population (left panel) and the whole extended sample (right panel).

The correlation found between $S_{V}^{\prime}$ and semi-major axis was first suggested by Vilas \& Smith (1985) and Harmann et al. (1987) and strongly supported by Lagerkvist et al. (1993) analyzing the spectra of a sample of D-type asteroids. On the other hand Carvano et al. (2003) did not find any correlation, analyzing a larger sample of featureless spectra. Nevertheless, their analysis was performed on a sample located mostly in the inner region of the asteroid belt, while our analysis, as well as Lagerkvist et al. (1993) uses a sample of objects located mostly in the outer belt. Note that we have found the correlation not only for the ACOs but also for the extended sample, including objects from NEO orbits to Trojan asteroids. This relationship may indicate a compositional difference, a reddening process more effective at larger distances, or a process that makes the spectrum less red at lower heliocentric distances (Nesvorny et al. 2005).

\subsubsection{Spectral gradient distribution}

The Spearman test gives information on the relationship between $S_{V}^{\prime}$ and several other parameters, but it does not give a clear view of the distribution of spectral gradients. This can be achieved by comparing the $S_{V}^{\prime}$ cumulative distribution for the different populations. In this section we will present the cumulative color distribution of the ACOs, the extended sample of featureless objects presented here, and the other two population for which we computed $S_{V}^{\prime}$ for a large number of objects, the Cybele and Trojan asteroids.

The cumulative distribution was chosen rather than a common histogram, due to the fact that it does not rely on the bin-size. The distribution of visible spectra gradients (Fig. 14) shows that the ACOs (dotted-dashed line) have an excess of objects with $S_{V}^{\prime}<1$ when compared with all the other samples. The Trojan asteroids (continous line) are, by far, the reddest sample, having few objects with $S_{V}^{\prime}<7$. On the other hand, the Cybeles (dashed line) seem similar to the ACOs for $1<S_{V}^{\prime}<10$ and a deficiency of objects with $S_{V}^{\prime}>10$. The median-mean values of the distributions are 3.527-4.037, 8.787-8.840, and 3.157-4.431 for the ACOs, Trojan and Cybele asteroids, respectively. For the extended sample the medianmean is 5.203-5.538. Note that all these values are given in units of $\% 0.1 \mu \mathrm{m}$.
The $S_{V}^{\prime}$ distribution of the ACOs is fairly similar to the Cybele asteroids, while very different from the Trojan asteroids, but, in any case, ACOs have a bluer distribution than Cybele and Trojan asteroids. The Trojan asteroids are redder than any of the other samples. Even if the color information about comets and Damocloids is mainly based on photometric measurements (e.g. Jewitt 2005), so the comparison is not straightforward, we should mention that the Damocloids present red spectral slopes with $S_{V}^{\prime}$ values between 5 and 17\%/0.1 $\mu \mathrm{m}$, and also that most comet nuclei (all but two cases in Jewitt 2005) and others recently observed (Abell et al. 2005; Campins et al. 2006) have also $S_{V}^{\prime}>5 \% / 1000$. Damocloids are very unlikely to be asteroids, they are probably dormant/dead Halley-type comets. Therefore, unless there is an unknown process that makes the surface of dormant comets bluer in the ACO population, the best candidates of being dormant/dead comets are the redder ones. Notice that this is the case of our ACOs with smaller $T$, which have a lower probability of an asteroidal origin and in more unstable orbits as discussed above. Also, the reddest ACOs have colors compatible with Trojan and Hilda asteroids (Dahlgren \& Lagherkvist 1995; Dahlgren et al. 1997; Jewitt 2005), and Trojan and Hilda asteroids can evolve onto JFC-like orbits (Levison \& Duncan 1997; Marzari et al. 1997; Di Sisto et al. 2005).

The $S_{V}^{\prime}$-distribution of the two sub-population of ACOs is shown in Fig. 14 The NEOs and non-NEOs show different distributions, the former beeing significantly redder. The median-mean values of the distributions are, 3.903-5.644, and 2.416-3.234 for the NEOs and non-NEOs, respectively. It is important to mention that the comparison was performed over different samples that cover different range in size and therefore are not definitive. Nevertheless in the common range of sizes, the difference stands.

\section{Conclussions}

In this paper we present visible and/or near-infrared spectra of 24 ACOs, 5 Hilda, 1 Trojan and 4 D-type inner main-belt asteroids, covering the $0.5-2.4 \mu \mathrm{m}$ spectral region, obtained using three different telescopes of the "Roque de los Muchachos Observatory" (Canary Islands, Spain): the $4.2 \mathrm{~m} \mathrm{WHT, \text {the }}$ $3.6 \mathrm{~m} \mathrm{TNG}$, and the $2.5 \mathrm{~m}$ NOT. 

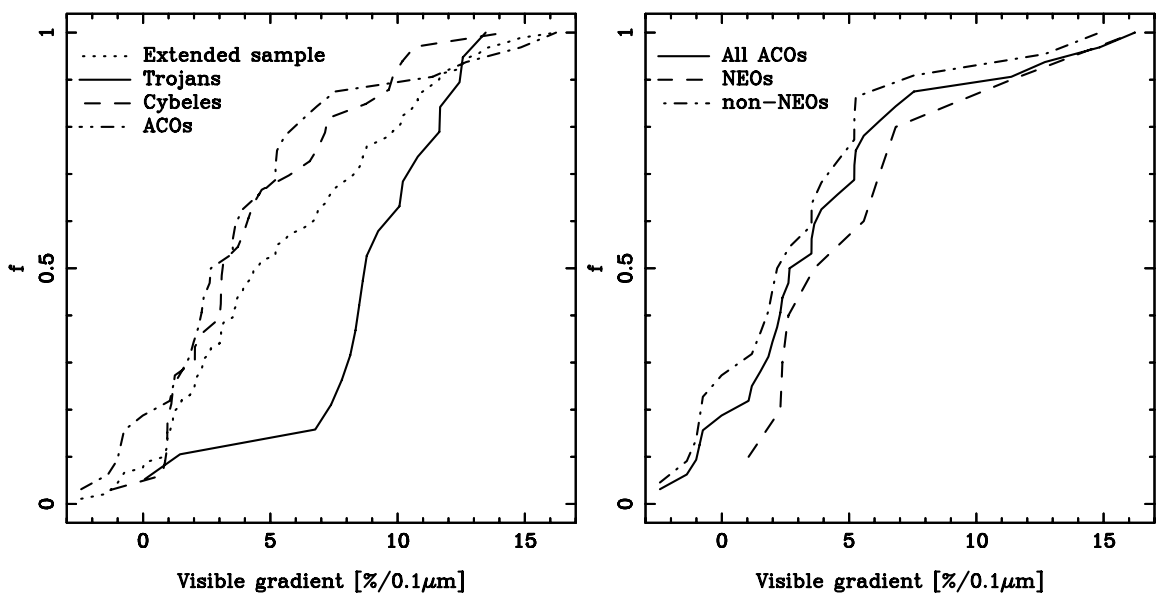

Fig. 14. Cumulative color distribution of the ACOs population, Cybele and Trojan asteroids and the whole extended sample is presented in the left panel. The right panel show the distribution of all ACOs and the considered individual populations (NEOs and non-NEOs).
We determined the taxonomic class of the observed objects using the taxonomy based on the visible $(0.3-1.1 \mu \mathrm{m})$ spectrum defined by Tholen \& Barucci (1989). We also included in the analysis the spectra of ACOs in the major visible spectroscopic surveys, the S3OS2 (Lazzaro et al. 2004 ) and the SMASS (Xu et al. 1995; Bus \& Binzel 2002). The final sample has 41 ACOs with visible and/or near-infrared spectrum. Most of the observed ACOs (34) have featureless spectrum, with colors that range from slightly bluish (B-type) to red (D-type), typical of outer belt asteroids and/or comet nuclei. Seven ACOs are with bands are unlikely to have a cometary origin: four S-type and three V-type. ACOs with bands (6 of 17, 35\%) belong to the NEO population (objects with perihelion distance $q<1.3 \mathrm{AU}$ ), while the non-NEO population of ACOs (objects with $q>1.3 \mathrm{AU}$ ) is almost devoid of asteroids with bands ( 1 with bands in $24, \sim 4 \%$ ). The abundance of objects with bands in the NEO sub-population of ACOs seem to indicate that it is composed of a significant fraction of asteroids dynamically scattered from the inner main belt. On the other hand, the non-NEO sub-population seems to be mostly replenished by dynamically scattered asteroids from the outer main belt and/or deactivated comet nuclei. Also all observed ACOs with $T<2.9$ are featureless, so the mechanism that drive inner main-belt asteroids to cometary orbits is only efficient in producing orbits with Tisserand very close to 3 .

We searched for any subtle features that could help to discriminate whether an ACO comes either from a cometary or an asteroidal source by comparing the spectra of the featureless ACOs with highest $\mathrm{S} / \mathrm{N}$ with the spectra of some Hilda, Trojan, Cybele asteroids, and D-type inner main-belt asteroids of similar size to that of the selected ACOs, and comet nuclei. We conclude that in the $0.5-2.0 \mu \mathrm{m}$ there are no subtle features that can be used to discriminate the cometary or asteroidal nature of the ACOs.

We performed an analysis to compare the spectral slope of featureless ACOs with that of the other populations dominated by featureless spectra. The featureless spectra presented in this paper and that of 18 Trojan and 33 Cybele asteroids selected from the S3OS2 and SMASS surveys were considered. The spectral slopes in the visible and near-infrared $\left(S_{V}^{\prime}\right.$ and $\left.S_{N}^{\prime}\right)$ of a total of 95 objects, including 34 ACOs, 19 Trojan, 33 Cybele, 5 Hilda and 4 D-type inner main-belt asteroids were obtained. We searched for correlations between the spectral slopes and the size (absolute magnitude) and orbital parameters $(T, a, e$, $i$ ) of all the featureless bodies, and the different populations separately: ACOs (NEO and non-NEO), Trojan, Hilda, and Cybele asteroids. The correlations found seem to relate dynamical and surface properties of ACOs. We found a strong anti-correlation between spectral slope and Tisserand parameter (with the reddest ACOs having the lowest $T$ ), and a weak correlation between spectral slope and $a$ and $e$. It is important to note that this relation is shared by both sub-populations of ACOs, NEOs and nonNEOs. The reddest ACOs have $2.7 \leq T$ and they are located in very unstable regions of the perihelion distance - Tisserand $(q-T)$ parameter space, therefore they should have been injected onto such orbits recently. On the other hand, bluer featureless ACOs (as well as with the majority of the ACOs with bands) are in a region with $(2.7<T \leq 3 ; 0.6<q<1.8 \mathrm{AU})$ a region that seems to be shared by bona fide (interlopers) asteroids and dormant/dead comets. The weak $S^{\prime}$ - $a$ relationship may indicate a compositional difference, a reddening process more effective at larger distances, or a process that makes the spectrum less red at lower heliocentric distances, while the $S^{\prime}-e$ arises from the relationship between $T$ and eccentricity.

The $S_{V}^{\prime}$ cumulative distribution of the featureless ACOs is "bluer" than that of Cybele and Trojan asteroids, but fairly similar to the Cybele asteroids one in the $1<S_{V}^{\prime}<10$ range. Considering also that the color distribution of Hilda asteroids is similar to that of Trojan asteroids (Dahlgren \& Lagerkvist 1995), we conclude that the color distribution of ACOs is different than that of outer belt asteroids. Also most comet nuclei have redder spectra than ACOs, with $S_{V}^{\prime}>5 \% / 1000$ (Jewitt 2002; Abell et al. 2005; Campins et al. 2006), and the best candidates of being Halley-type dormant comets, the Damocloids, present redder spectral slopes than ACOs, with $5<S_{V}^{\prime}<17 \% / 0.1 \mu \mathrm{m}$ (Jewitt 2005). Thus the color distribution of ACOs argues against a pure cometary and/or outer main-belt origin. Notice that the reddest ACOs (with colors compatible with Trojan and Hilda asteroids, Damocloids and comet nuclei) are those with a lower probability of an asteroidal origin $(T<2.7)$ and also have comet-like albedos $\left(p_{V}<0.075\right.$, Fernandez et al. 2005). As shown, the region with $(2.7<T \leq 3 ; 0.6<q<1.8 \mathrm{AU})$ is "contaminated" by bona fide asteroids scattered from the inner main belt. The inner main-belt has a larger abundance of "bluer" (B- and C-type) featureless asteroids than in the outer belt and comet population, thus this contamination of inner main-belt featureless asteroids is probably the cause of the color distribution of ACOs. Finally the NEOs and non-NEOs population show different $S_{V}^{\prime}$ cumulative distributions, the former being redder. In conclusion, ACOs with $T<2.7$ are the best candidates of being dead or dormant comets because of their surface and dynamical properties, and deserve a more detailed study. 
Acknowledgements. Based on observations made with the Italian Telescopio Nazionale Galileo (TNG) operated on the island of La Palma by the Fundación Galileo Galilei of the INAF (Instituto Nazionale di Astrofisica) at the Spanish Observatorio del Roque de los Muchachos of the Instituto de Astrofísica de Canarias. We thanks the referee, Dr. E. Howell for her useful comments. We wish to thank Dr. E. Howell for her comments that helped us to improve the manuscript. A.A.C. and D.L. would like to thank the Conselho Nacional de Desenvolvimento Cientifico e Tecnologico (CNPq) and the Fundacao de Amparo a Pesquisa do Estado do Rio de Janeiro (FAPERJ), that supported this work through diverse fellowships and grants. A.A.C. would like to thanks Conselho Nacional de Desenvolvimento Científico e Tecnólogico $(\mathrm{CNPq})$ - Brazil, that supported this work via Ph.D. fellowship. H.C. aknowledges the support from grants from the National Science Founation and NASAÕ Planetary Astronomy program.

\section{References}

Abell, P., Fernandez, Y., Pravec, P., et al. 2005, Icarus, 179, 174

Alvarez-Candal, A., \& Roig, F. 2004, in Dynamics of Populations of Planetary Systems, ed. Z. Knežević, \& A. Milani (Cambridge: Cambridge Univ. Press), 205

Alvarez-Candal, A., \& Licandro, J. 2006, A\&A, 458, 1007

Barucci, M. A., Doressoundiram, A., \& Cruikshank, D. P. 2004, in Comets II, ed. M. Festou, H. U. Keller, \& H. A. Weaver (Tucson: Univ. of Arizona Press), in press

Bell, J., Owensby, P., Hawke, B., \& Gaffey, M. 1988, in Abstracts of the Lunar and Planetary Sci. Conf., 19, 57

Binzel, R., Rivkin, A. Stuart, J., et al. 2004, Icarus, 170, 259

Bottke, W. F., Morbidelli, A., Jedicke, R., et al. 2002, Icarus, 156, 399

Bus, S. J., \& Binzel, R. P. 2002, Icarus, 158, 106

Campins, H., Ziffer, J., Licandro, J., et al. 2006, AJ, 132, 1346.

Carvano, J.M., Mothé-Diniz, T., \& Lazzaro, D., 2003, Icarus, 161, 356

Colina, L., \& Bohlin, R. 1997, AJ, 113, 1138

di Sisto, R., Brunini, A., Dirani, L., \& Orellana, R. 2005, Icarus, 174, 81

Dahlgren, M., \& Lagerkvist, C. 1995, A\&A, 302, 907

Dahlgren, M., Lagerkvist, C., Fitzsimmons, A., Williams, I., \& Gordon, M. 1997, A\&A, 323, 606

Fernández, J., Gallardo, T., \& Brunini, A. 2002, Icarus, 159, 358

Fernandez, Y., Jewitt, D., \& Sheppard, S. 2005, AJ, 130, 308

Gil-Hutton, R. 2002, Plan. Space Sci., 50, 57
Gradie, J. C., Chapman, C. R., \& Tedesco, E. F. 1989, Distribution of taxonomic classes and the compositional structure of the asteroid belt, in Asteroids II, ed. R. Binzel, T. Gehrels, \& M. Shapley Matthews (Tucson: Univ. of Arizona Press), 316

Hartmann, W., Cruickshank, D., \& Degewij, J. 1987, Icarus, 52, 377

Hsieh, H. H., \& Jewitt, D. 2006, Science, 312, 561

Jewitt, D. 2002, AJ, 123, 1039

Jewitt, D. 2005, AJ, 129, 530

Kresák, L. 1979, In Asteroids, ed. T. Gehrels (Tucson, Univ. Arizona Press), 289

Lagerkvist, C.-I., Fitzsimmons, A., Magnusson, P., \& Williams, I. P. 1993, Mon. Not. Astron. Soc., 260, 679

Landolt A. 1992, AJ, 104, 340

Lazzaro, D., Angeli, C. A., Carvano, J. M., et al. 2004, Icarus, 172, 179

Levison, H. F., \& Duncan, M. J. 1997, Icarus 127, 13

Licandro, J., Ghinassi, F., \& Testi, L. 2002a, A\&A, 388, L9

Licandro, J., Campins, H., Hergenrother, C., \& Lara, L. M. 2002b, A\&A, 398, L45

Licandro, J., de León, J., Pinilla, N., \& Serra-Ricart, M. 2006, AdSpR, 38, 1991

Licandro, J., Campins, H., Mothé-Diniz, T., Pinilla, N., \& de León, J. 2007, A\&A, 461, 751

Luu, J., \& Jewitt, D. 1996, AJ, 112, 2310

Marzari, F., Scholl, H., Tomasella, L., \& Vanzani, V. 1997, Planet. Space Sci., 45,337

Mothé-Diniz, T., Lazzaro, D., Carvano, J. M., \& Florczak, M. 2000, Icarus, 148, 497

Mothé-Diniz, T., Carvano, J. M., \& Lazzaro, D. 2003, Icarus, 162, 10

Nesvorny, D., Jedicke, R., Whiteley, R., \& Ivezic, Z. 2005, Icarus, 173, 132

Oliva, E. 2000, MemSAIt, 71, 861

Opik, E. J. 1963, Irish A.J. 6, 93

Press, W. H., Teukosky, S. A., Vetterling, W. T., \& Flannery, B. P. 1992, Numerical Recipes, 2nd edn (Cambridge, UK: Cambridge Univ. Press)

Rickman, H., Fernández, J., \& Gustafson, B. 1990, A\&A, 237, 524

Tancredi, G., Fernández, J., Rickman, H., \& Licandro, J. 2006, Icarus, 182, 527

Thébault, P., \& Doressoundiram, A. 2003, Icarus, 162, 27

Tholen, D. J. 1984, Asteroid taxonomy from cluster analysis of photometry, Ph.D. Thesis

Tholen, D. J., \& Barucci, M. A. 1989, in R. Binzel, T. Gehrels, \& M. Shapley Matthews, Asteroids II (Tucson: Univ. of Arizona Press), 298

Vilas, F., \& Smith, B. 1985, Icarus, 64, 503

Xu, S., Binzel, R. P., Burbine, T. H., \& Bus, S. J. 1995, Icarus, 115, 1 